\title{
PATENT AND LICENSING STRATEGY - as a control and analytical algorithm for the readiness of smart technology for mass production and integrated marketing
}

\author{
Yerzhan Kusparmakov \\ Corresponding member of International Informatization Academy, Professional \\ Member of the New York Academy of Sciences, Master of Business \\ Administration, Head of Product management at SAMSUNG Electronics \\ e-mail:kusparmakov@gmail.com
}

\begin{abstract}
Recently, several new, in many ways, fundamentally new directions for the development of innovative high-tech products, which are unequivocally products of mass demand, have taken shape systematically.

Such innovative areas include those named and classified as - smart products and technologies, products such as - smart design, like smart medical technologies, equipment and instruments, which certainly include medical equipment and devices with effective mobile applications, smart transport and smart transportation, smart manufacturing and smart manufacturing equipment, including special technological equipment with digital program control and the most advanced processing centers and flexible manufacturing cells.
\end{abstract}

Keywords: Patent, Licensing, Smart technology, Mass production, Smart manufacturing, Storage media, Data encryption, Start-up a business in the USA

В последнее время системно оформились несколько новых, во многом - принципиально новых направлений развития инновационных продуктов высоких технологий, которые однозначно являются продуктами массового спроса.

К таким инновационным направлениям можно отнести, названные и классифицированные как - умные продукты и технологии, такие продукты как - умный дизайн, как умные медицинские технологии, оборудование и инструментарий, безусловно включающие в себя и медицинскую технику и приборы с эффективными мобильными приложениями, умный транспорт и умное транспортирование, умное производство и умное производственное оборудование, включая и специальное технологическое оборудование с цифровым программным управлением и наиболее совершенные обрабатывающие центры и гибкие производственные модули.

Сюда же входят и системы подготовки - умной рабочей силы (Smart workforce development). 
Рассмотрим весь этот процесс на примере внедрения модификаций мобильного защитного кодирования цифровых внешних носителей информации.

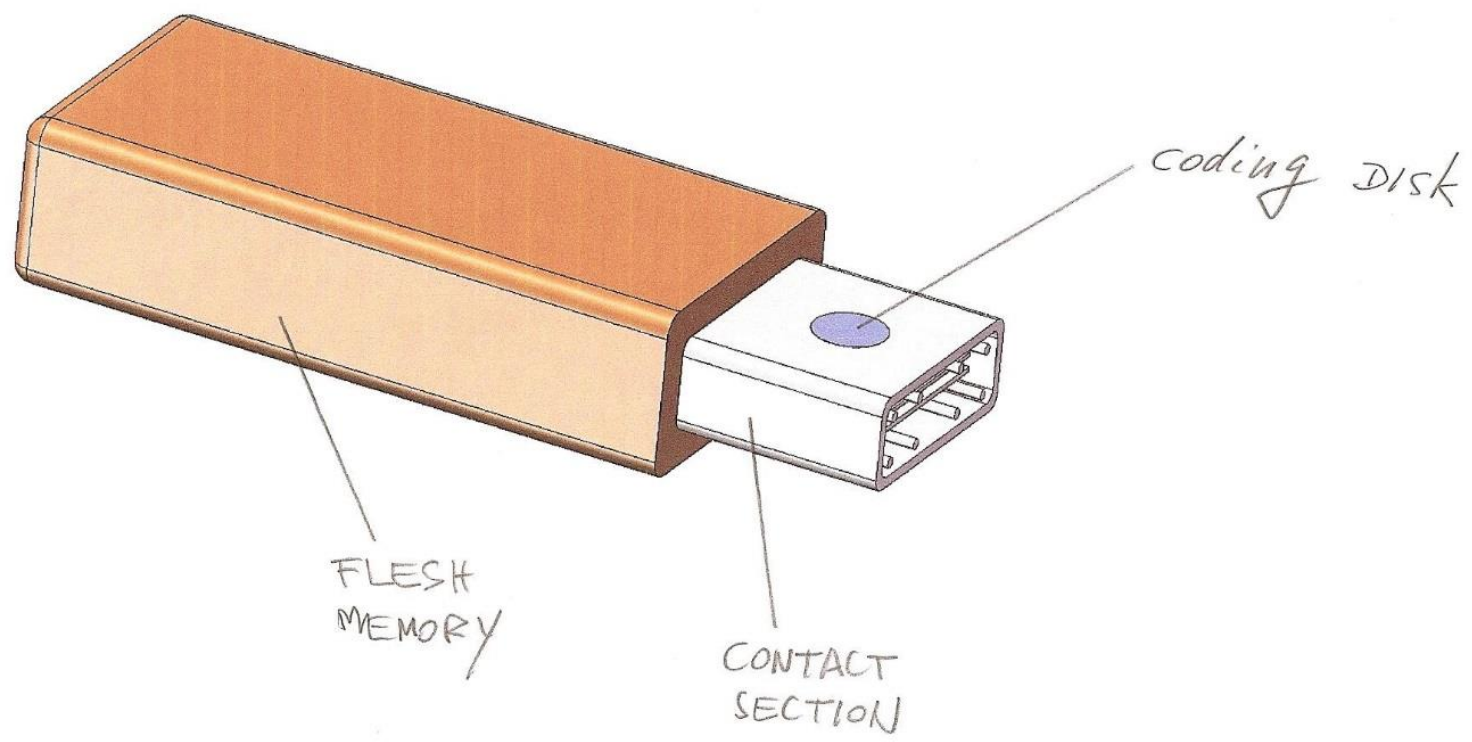

Рисунок 1, - на рисунке представлен пример такого внешнего носителя информации, модифиџированный инновационной системой кодирования

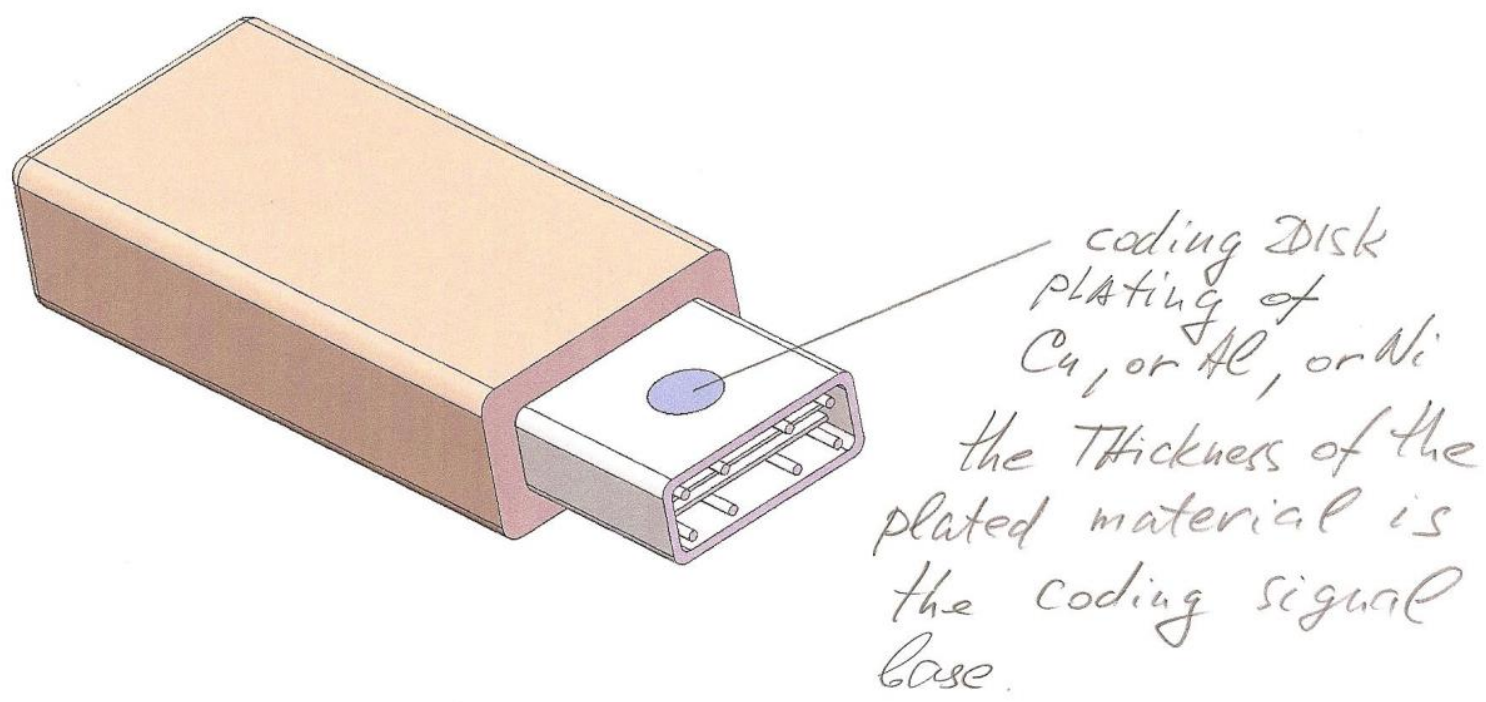

Рисунок 2, - на рисунке также представлен пример такого внешнего носителя информации, модифицированный инновационной системой кодирования

Принцип системы кодирования и использованные для её построения материалы, и их сочетания также показаны на рисунке. 
Решение такого кодирующего и декодирующего узла, при всей своей простоте, позволяет получить максимальную точность идентификации кода, так как указанная точность определяется точностью толщины кодирующего или декодирующего диска.

Имеется как минимум два метода изготовления и установки на корпусе этого диска, первый метод, - это метод изготовления кодирующего диска из прецизионной фольги, второй метод - это нанесение прецизионного электрохимического покрытия в проточке, выполненной в корпусе для кодирующего диска.

\section{Общая информация}

Bсе проекты этой группы технических решений базируются на одном методе кодирования и последующей идентификации записи кодирующего элемента.

Сущность принципа состоит в нанесении на защищаемый объект кодирующего покрытия или его технологического эквивалента и последующего измерения толщины этого покрытия, определяющего совпадение или не совпадение результатов измерения с кодом.

При совпадении полученного результата с установленным происходит положительная идентификация кодирующего элемента, при не совпадении, - происходит отрицательная идентификация и остановка или блокирование рабочего цикла оборудования или потребителя информации, например, - компьютера.

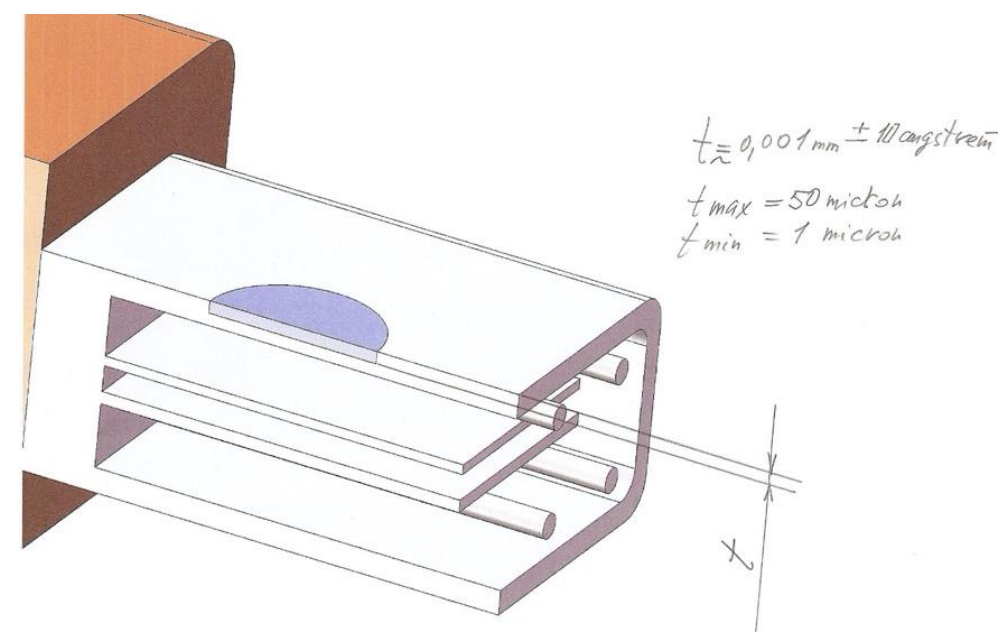

Рисунок 3, - на рисунке также представлен пример такого внешнего носителя информации с указанием технических деталей, модифищированных инновационной системой кодирования 
В сечении показана наиболее критичная величина, определяющая точность кодирования или декодирования, - толщина диска - t.

Минимальная толщина может быть равной - 1 микрону, максимальная толщина - 50 микронам.

\section{Дополнительные технологические особенности}

Технологически вопросы нанесения специальных покрытий решены, и эта технология была многократно проверена на аналогичных задачах, связанных с контролем толщины плёнок на панелях солнечных батарей и в традиционном полупроводниковом производстве.

Дополнительные особенности и возможности применения технологии, применительно к новым условиям, возникшим на рынке носителей и накопителей информации в течении последнего года с учётом возникших новых направлений в технических решениях и технических системах любых уровней в конечном счёте приводящих к синтезу так называемых умных технологий и комплексных технических систем с элементами искусственного интеллекта и искусственными нейронными сетями. 


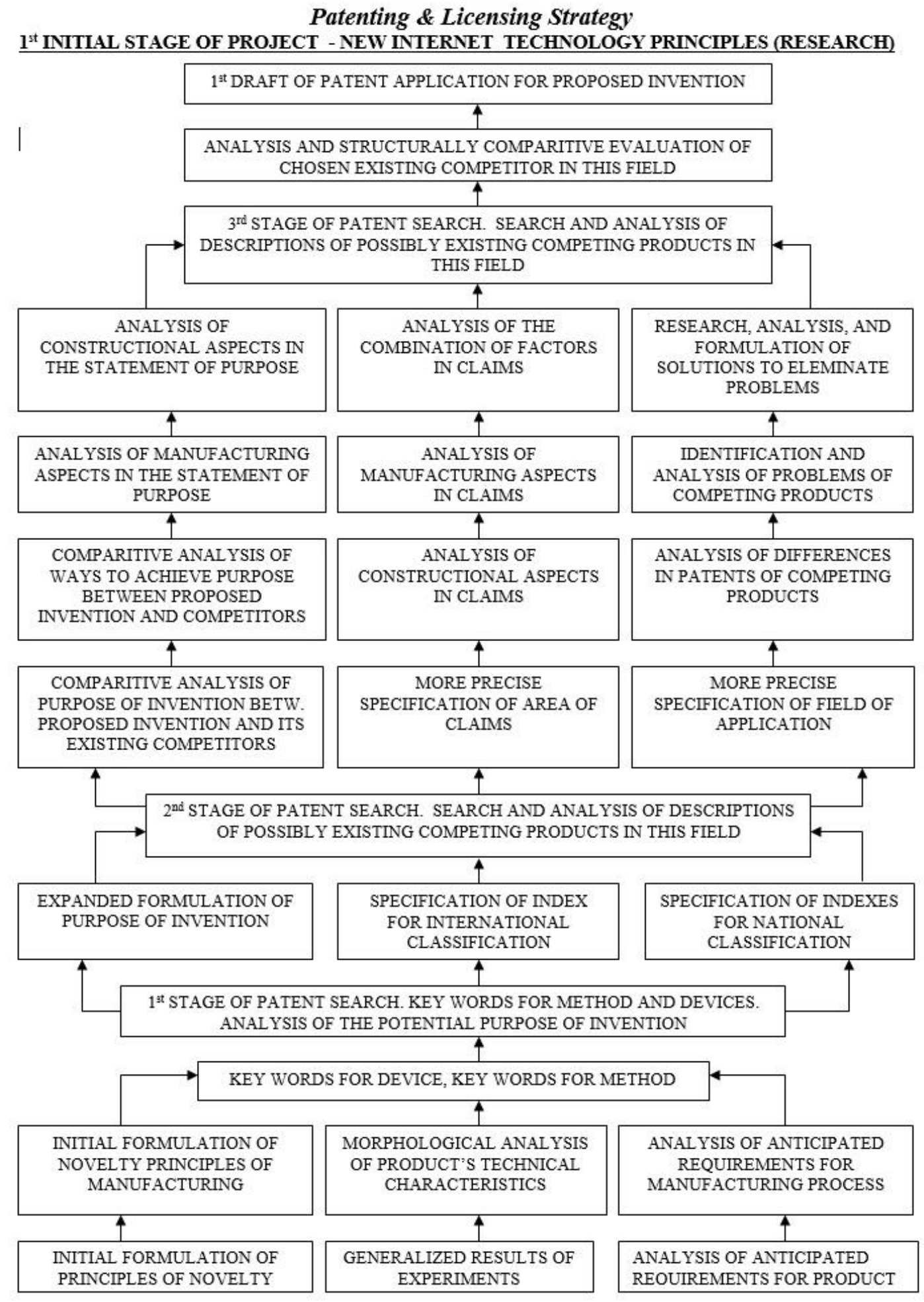

Рисунок 4, - на рисунке представлен алгоритм применения патентной и лищензионной стратегии на начальных стадиях развития проекта 
Patenting \& Licensing Strategy

$2^{\text {nd }}$ INITIAL STAGE OF PROJECT - Codification Technology Principles and Internet integrated solutions DEALS WITH CORE TECHNOLOY THAT RELATES TO BASE TECHNOLOGY AND CONSTRUCTION PRINCIPLES OF PRODUCT ("BASE"), AS OPPOSED TO A SPECIFIC APPLICATION OF THE PRODUCT

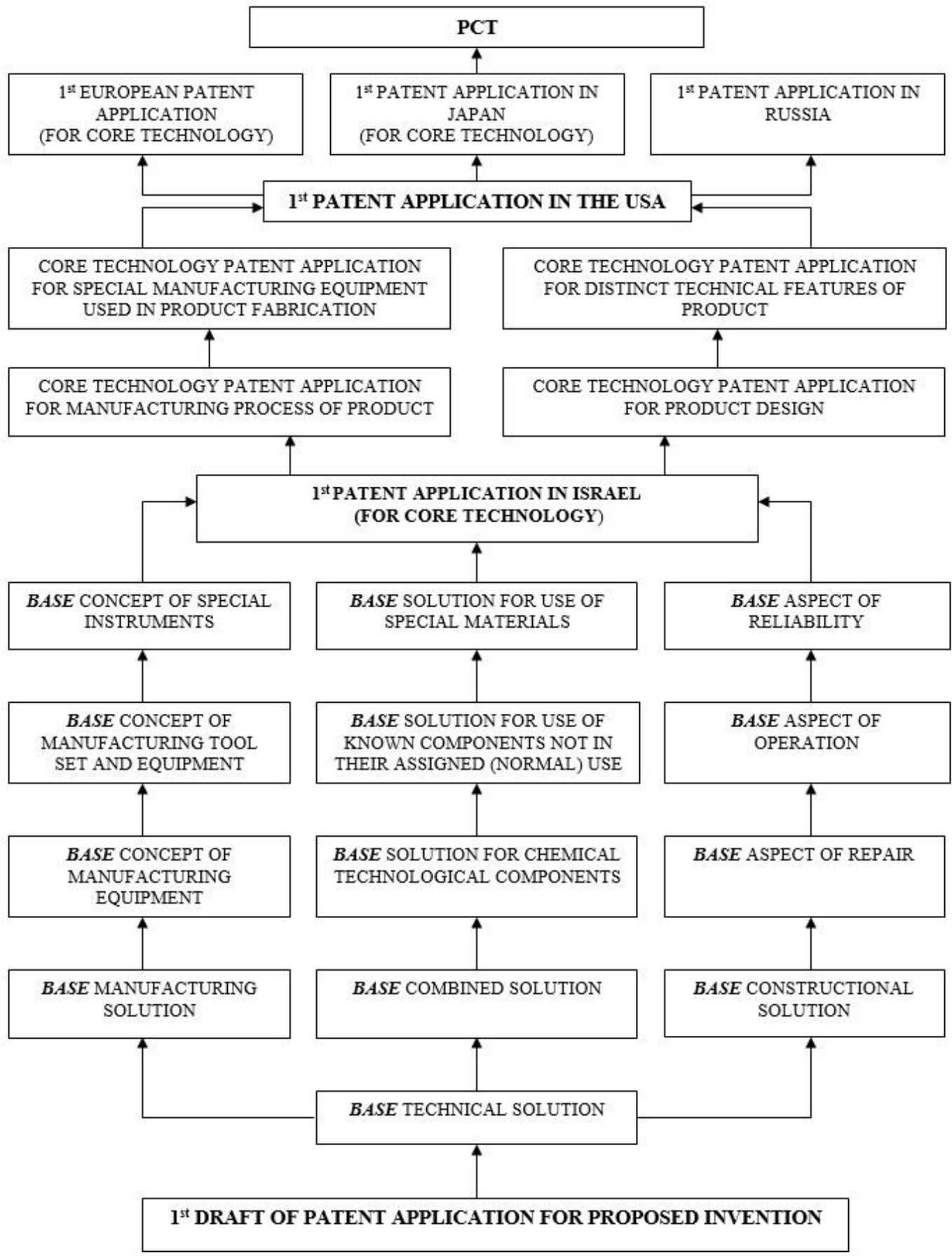

Рисунок 5, - на рисунке также представлен алгоритм применения патентной и лицеензионной стратегии на последующих стадиях развития проекта 


\section{Patenting \& Licensing Strategy}

Codification Technology Principles, Coder-Encoder system and Internet solutions and interfaces $3^{\text {rd }}$ INITIAL STAGE OF PROJECT (RELATING TO SPECIFIC APPLICATION)

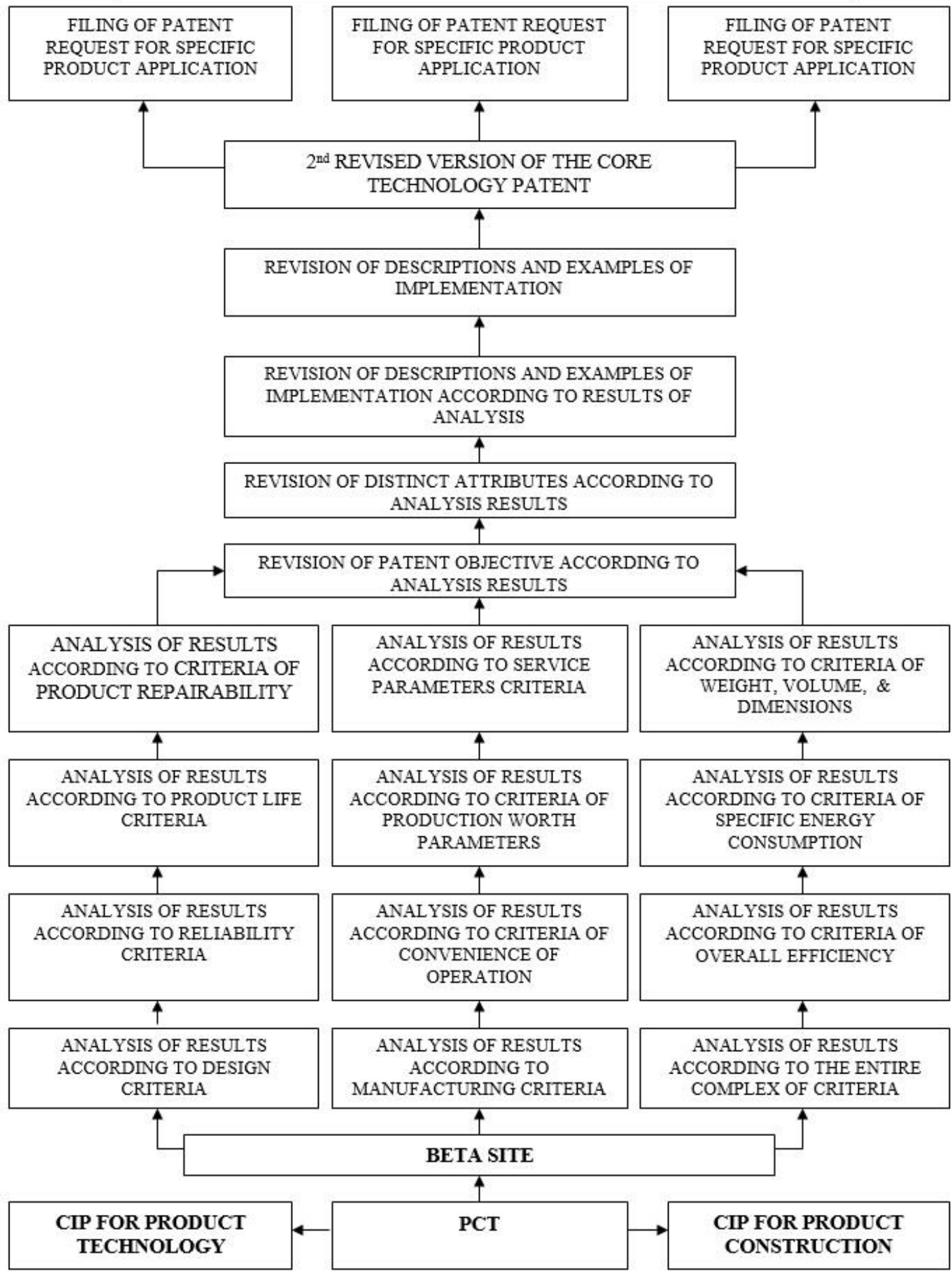

Рисунок 6, - на рисунке представлен алгоритм применения патентной и лицензионной стратегии на средних стадиях развития проекта 
Patenting \& Licensing Strategy

STAGES: DETAIL DESIGN FOR MASS PRODUCTION of CODIFICATION TECHNOLOGY PRINCIPLES and CODER-ENCODER systems FOR Internet solutions , - COMPONENTS PRELIMIINARY TESTING DOCUMENTATION FOR MASS PRODUCTION

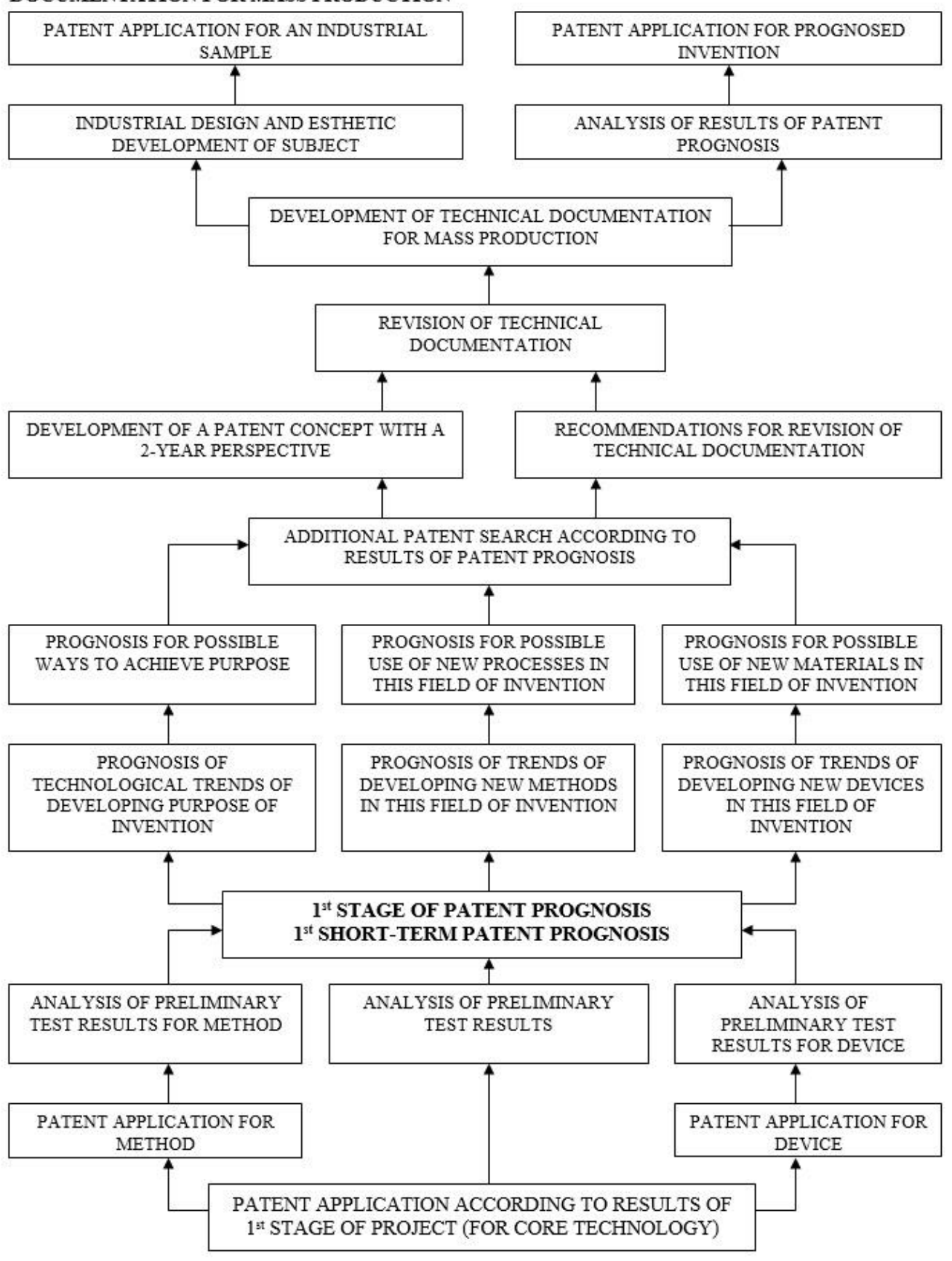

Рисунок 7, - на рисунке представлен алгоритм применения патентной и лищензионной стратегии на стадиях дизайна для массового производства 
Patenting \& Licensing Strategy

Codification Technology Principles, Coder-encoder system and Internet new solutions and interfaces STAGES:
TRIAL RUN OF MASS PRODUCTION
PRODUCT ACCEPTANCE TESTING
FULL SCALE MASS PRODUCTION

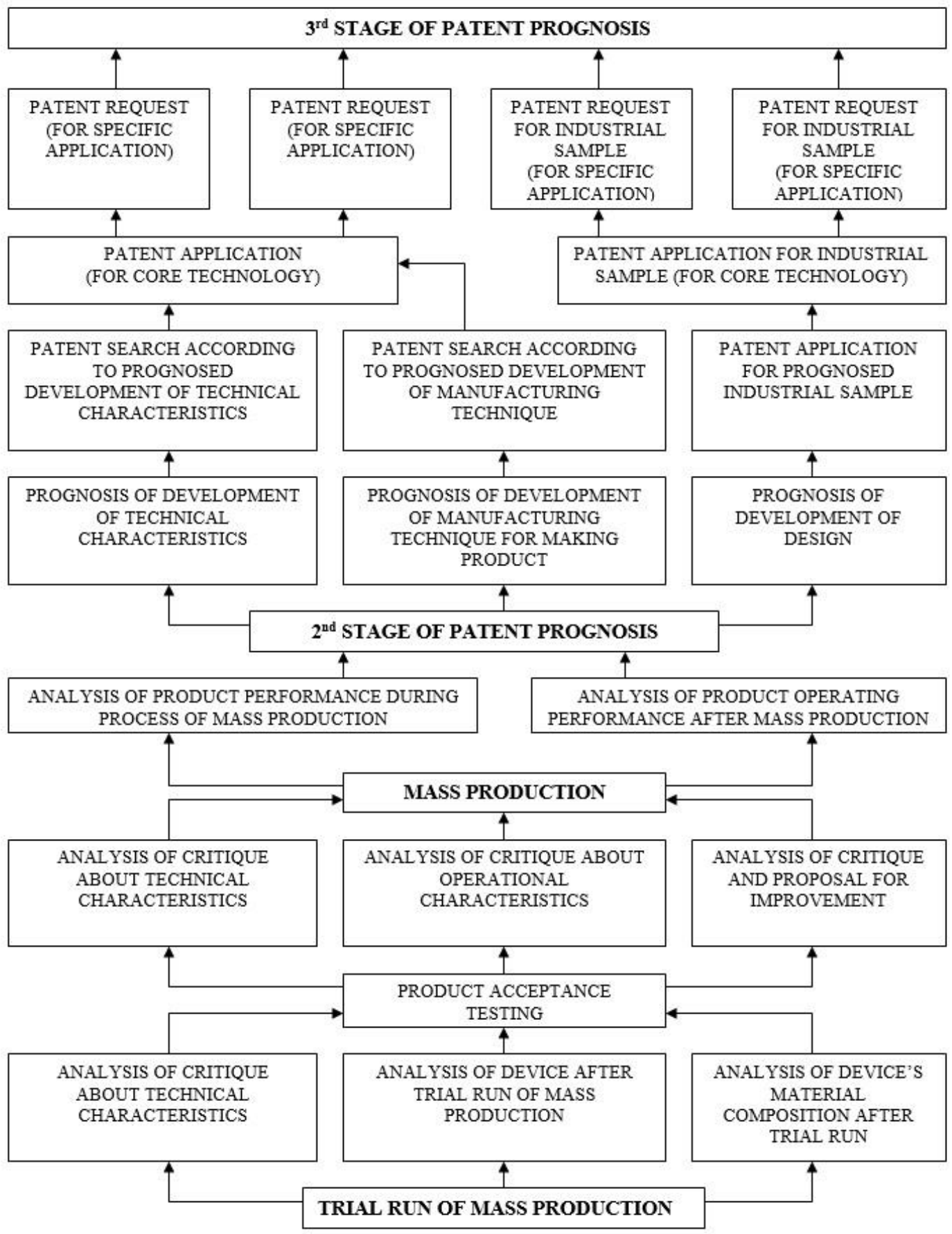

Рисунок 8, - на рисунке представлен алгоритм применения патентной и лищензионной стратегии на стадиях массового производства, этапах приёмных и 
квалификаџионных испьтаний и после корректировки по их результатам переход к полномасштабному массовому производству

Patenting \& Licensing Strategy

CODIFICATION TECHNOLOGY PRINCIPLES in Internet solutions, programs and interfaces STAGES: MASS PRODUCTION ACTIVE MARKETING PHASE PRODUCT IMPROVEMENT

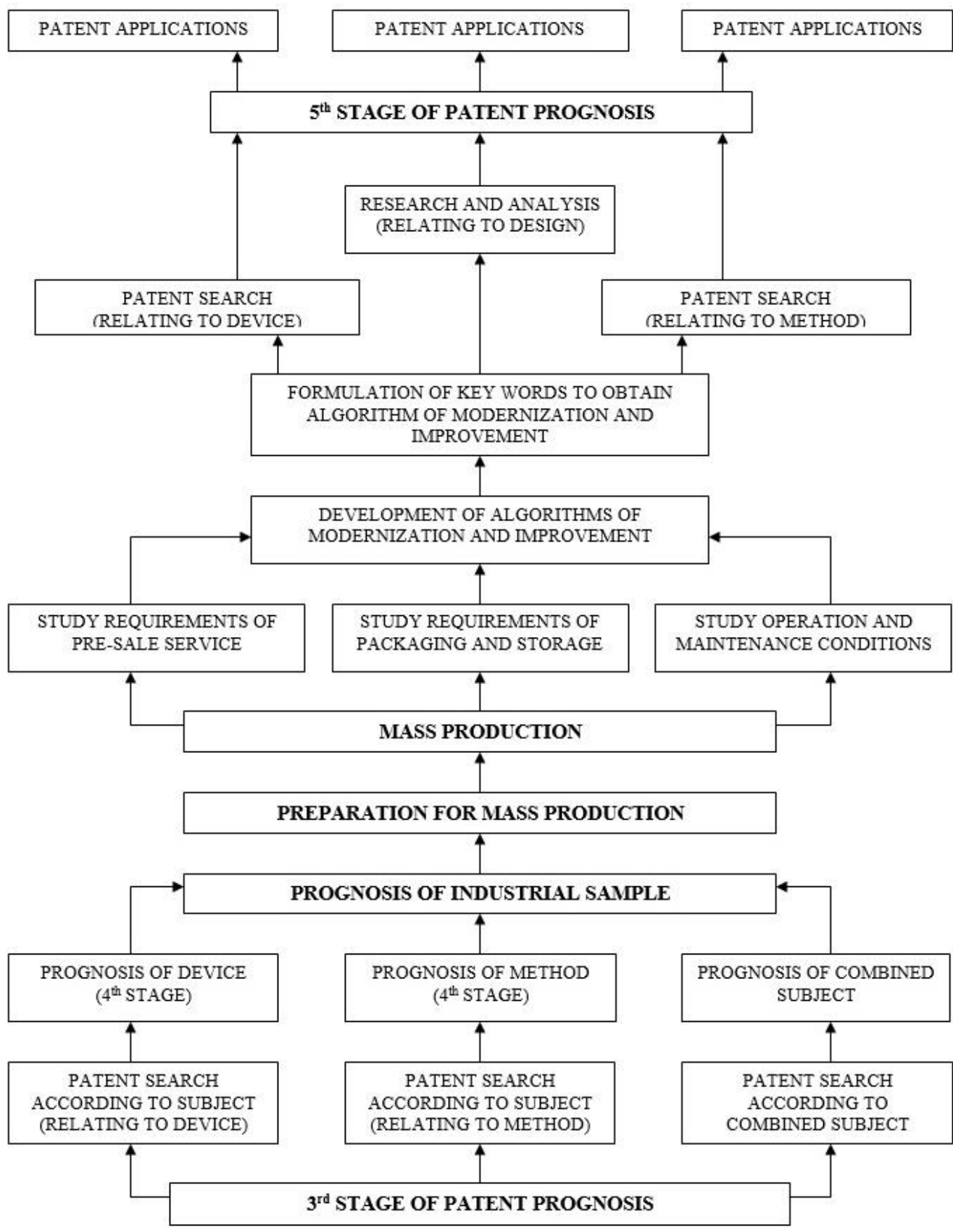

Рисунок 9, - на рисунке представлен алгоритм формирования и внедрения патентной и лицензионной стратегии на этапах развития проекта, - таких как, - массовое производство, активная фаза комплексного маркетинга и последующий после их и 
параллельно с ними интегративный этап оптимизации и модификации продукта с приданием продукту свойств и характеристик, - умного продукта, произведённого по

умной технологии, на умном оборудовании, с применением умных материалов и компонентов, при использовании умной рабочей сильв в рамках умной

производственной инфраструктурь

Ocобое внимание (в соответствии с алгоритмом) уделяется патентному прогнозированию в разрезе устройства, в разрезе - методов, в разрезе комбинированного и интегрированного объекта, по возможности с применением во всех составных частях инфраструктуры продукта, - элементов искусственного интеллекта с искусственными нейронными сетями.

По опыту маркетинга после реализации всех аспектов и особенностей массового производства нового продукта, в алгоритм внедряются аналитические требования к всем элементам системы обслуживания процесса маркетинга, - изучение предпродажного сервиса, изучение требований к упаковке и хранению, а также изучение требований и условий (особенно технических требований и технических условий) к порядку и методике ремонта и обслуживания.

Bce эти виды активности предусматривают интегративное сочетание всех вышеуказанных аспектов и на их базе возможное формулирование целей и задач по оптимизации и дальнейшему развитию проекта.

\section{Комментарии к алгоритмам (показанным на рисунках выше)}

Рисунок 4, - на рисунке представлен алгоритм применения патентной и лицензионной стратегии на начальных стадиях развития проекта.

Характерные вопросы, решаемые по этому фрагменту алгоритма:

○ формулировка общей новизны продукта;

○ формулировка общей эксплуатационной характеристики продукта;

○ формулировка технической характеристики продукта;

○ формулировки регламента производства продукта;

○ формулировка исходных технических требований к продукту и его производству;

○ формулировка требований к патентному поиску;

○ формулировка технической характеристики продукта и её потенциала сравнительной новизны и потенциала на последующие 5, 10, 15 лет.

Рисунок 5, - на рисунке также представлен алгоритм применения патентной и лицензионной стратегии на последующих стадиях развития проекта. 
Начало алгоритма - формирование первого варианта патентной аппликации по новому продукту с учётом всех особенностей и результатов всех видов патентного поиска, а также исследований рынка.

Завершение алгоритма - формирование и подача международной патентной заявки с учётом всех результатов исследований рынка и его требований к новому продукту.

В отработке алгоритма постоянно анализируется характер базовых концептуальных решений по всем характеристикам и свойствам нового продукта.

Рисунок 6, - на рисунке представлен алгоритм применения патентной и лицензионной стратегии на средних стадиях развития проекта.

Начало алгоритма - формирование и подача международной патентной заявки с учётом всех результатов исследований рынка и его требований к новому продукту, а также развитие проекта нового продукта и его приложений в формате - программа, система и ассоциированный метод по методу - CIP, - continuation in part.

Системный анализ результатов опытно промышленной эксплуатации и всех видов испытаний.

Завершение алгоритма - подготовка и подача системных заявок на предполагаемые изобретения, по материалам опытно-промышленной эксплуатации, по результатам испытаний и по результатам системного анализа всех полученных результатов этого этапа производства и реализации проекта нового продукта.

Рисунок 7, - на рисунке представлен алгоритм применения патентной и лицензионной стратегии на стадиях дизайна для массового производства.

Начало алгоритма - подготовка и подача системных заявок на предполагаемые изобретения, по материалам опытно промышленной эксплуатации, по результатам испытаний и по результатам системного анализа всех полученных результатов этого этапа производства и реализации проекта нового продукта.

Завершение алгоритма - подготовка материалов и подача аппликации на получение промышленного образца на новый продукт.

Кроме этого, в этот алгоритм вошли все стадии и этапы разработки технической документации, начиная с этапов технического задания, технического предложения, эскизного проекта, технического проекта, рабочего проекта, корректировок документации по результатам испытаний и опытно промышленной эксплуатации.

Также в этот алгоритм вошли этапы патентного поиска по массивам мировой информации с учётом результатов испытаний и их структурного анализа. 
Рисунок 8, - на рисунке представлен алгоритм применения патентной и лицензионной стратегии на стадиях массового производства, этапах приёмных и квалификационных испытаний и после корректировки по их результатам переход к полномасштабному массовому производству.

Начало алгоритма - массовое производство нового продукта

Завершение алгоритма - 3 этапа патентного прогнозирования, для выявления направлений оптимизации нового продукта.

\section{Теория решения изобретательских задач (ТРИЗ) и Алгоритмы решения изобретательских задач (АРИЗ)}

Теория решения изобретательских задач и все известные её производные Алгоритмы решения изобретательских задач были созданы в стране СССР и в период с 1945 по 1947 года, где коммерциализации инновационных решений не придавалось особого значения, можно даже сказать, что на определённом этапе создания Теории и Алгоритма решения изобретательских задач вопросы коммерциализации преднамеренно игнорировались в пользу чисто технологических вариантов инновационных решений, абсолютно оторванных от реальной экономики и в большинстве случаев от реальной жизни.

В результате такой близорукой и однобокой организационной модели развития инновационного процесса, имевшей место в это время, изобретатели, выросшие и воспитанные на классических приёмах и принципах ТРИЗ и АРИЗ оказались совершенно не подготовленными к особенностям и приёмам конкурентной борьбы в условиях современного общества с свободной конкурентной экономикой, особенно при постепенном переходе к системным умным технологиям, особенно применяющим элементы искусственного интеллекта и искусственные нейронные сети.

Они готовы и любят изобретать, но не готовы и не умеют заработать на своих изобретениях, чтобы получить достойную компенсацию за свой талант и творческий труд.

Особенно важно оценить необходимость начала инновационного процесса и понять, а ещё лучше рассчитать все возможные варианты развития событий в процессе коммерциализации.

Для этой цели мы и находим целесообразным применить систему оценочных алгоритмических критериев и таблиц в виде аналитического оценочных листов, алгоритма патентной и лицензионной стратегии, состоящего из 6 групповых элементов оценки критериев и параметров возникшей инновационной идеи или инициативы и процесса их развития и реализации. 
В связи с появлением новых форматов записи и чтения на оптических носителях информации с использованием голубых лазеров, и в связи с началом производства многослойных оптических дисков, базирующихся на этой же технологии, предложенные принципы и технические решения по защитному кодированию приобрели ещё большее значение, так как количество записанной информации на каждом диске увеличивается и отсутствие защиты приводит к всё большим потерям секретных или конфиденциальных данных.

В дополнение к уже переданной информации необходимо указать возможности по кодированию каждого слоя в многослойных дисках , при котором кодируется каждый уровень слоёв записи, что является существенным усовершенствованием системы форматирования оптического носителя информации в трёхмерном выражении и является средством обеспечения (для особо важной и секретной информации) локального избирательного кодирования информации в пределах одного диска.

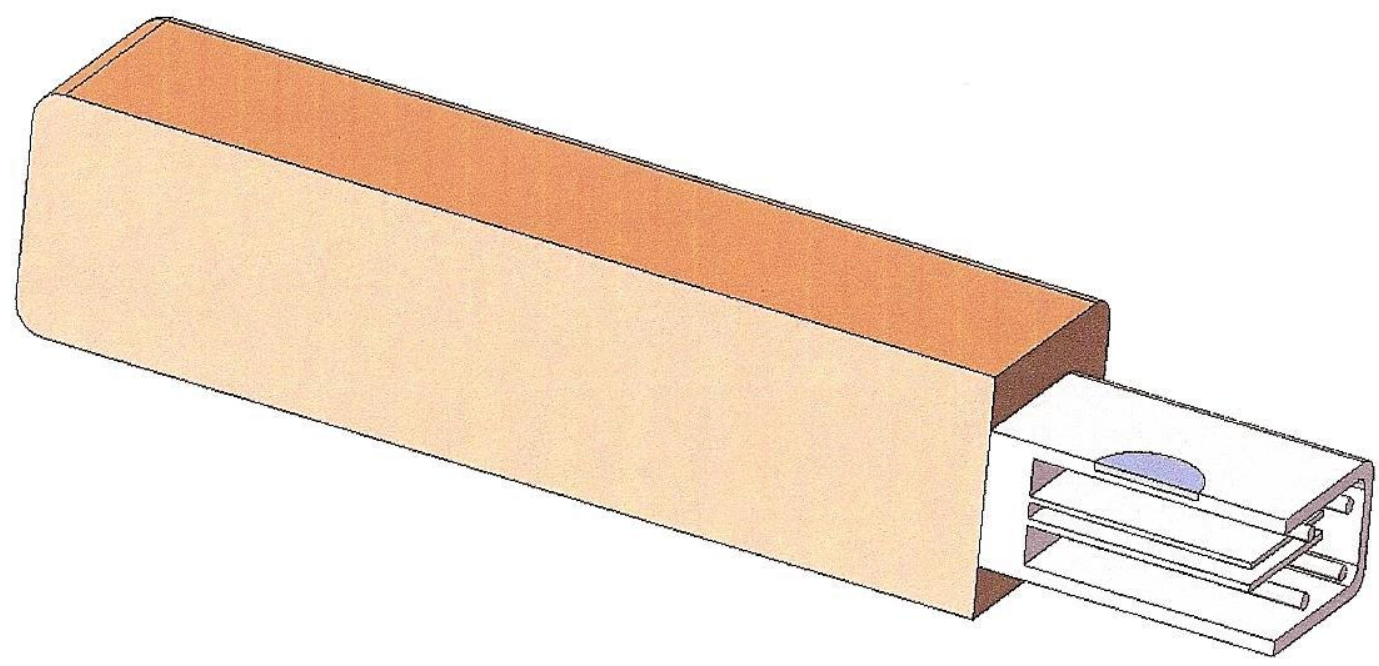

Рисунок 10, - на рисунке показана модель носителя информации в осевом сечении 


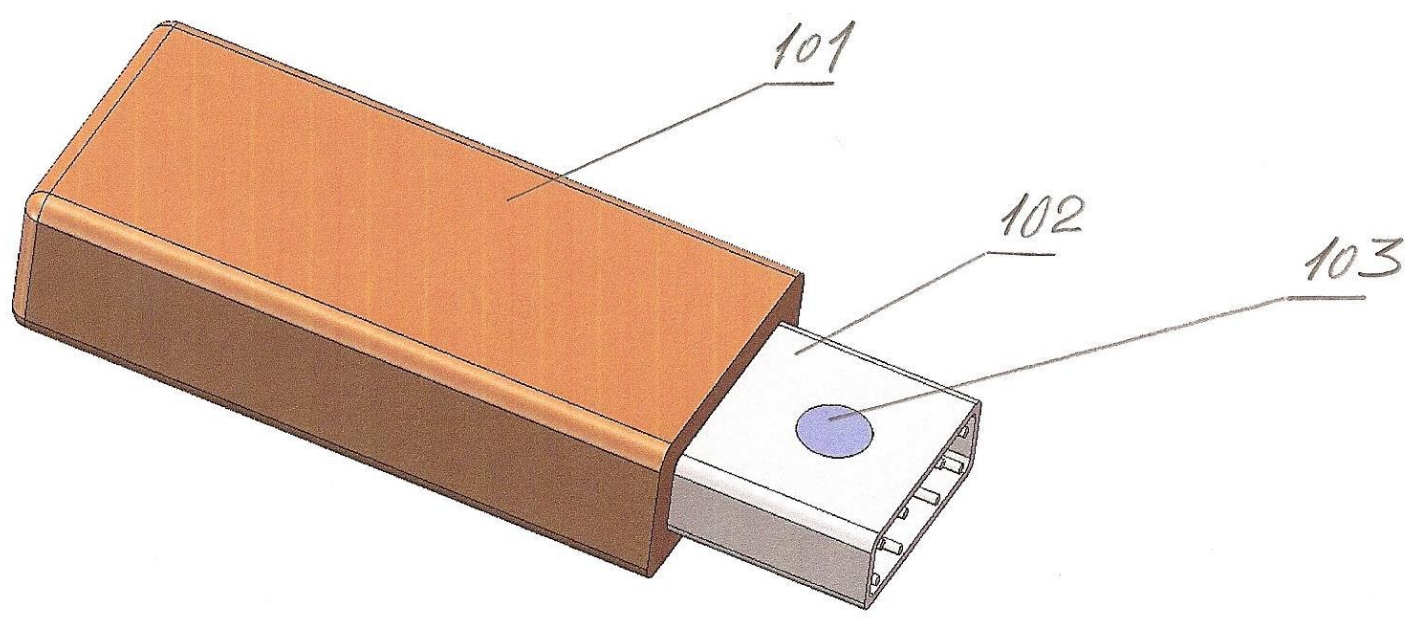

Рисунок 11, - на рисунке показана трёхмерная модель носителя информащии

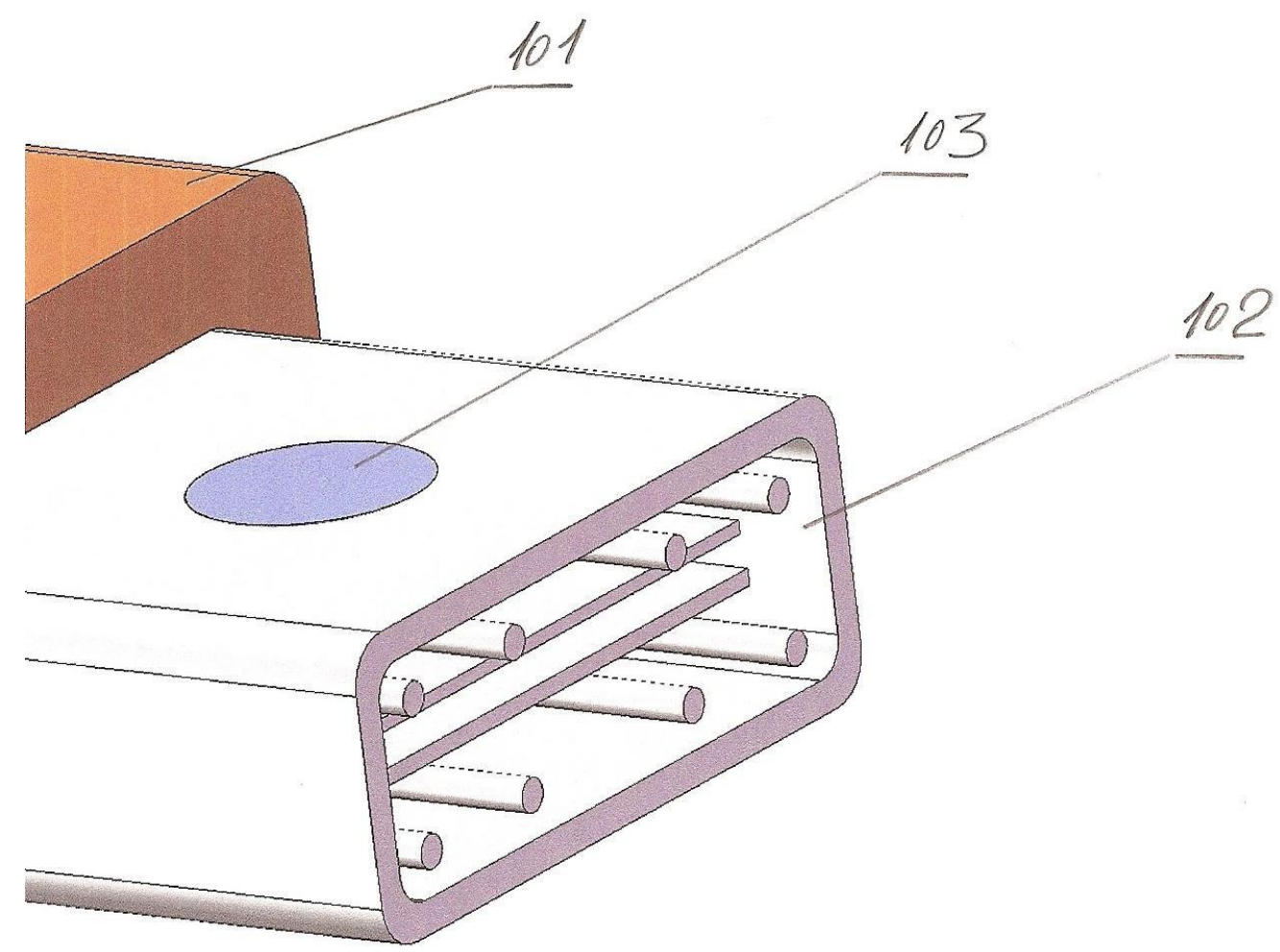

Рисунок 12, - на рисунке показан фрагмент трёхмерной модели носителя информации в районе плоского штекера с кодирующим и декодирующим диском

Цифрами на рисунке 11 и 12 обозначены:

○ 101, - корпус носителя информации; 
○ 102, - плоский штекер;

○ 103, - кодирующий диск.

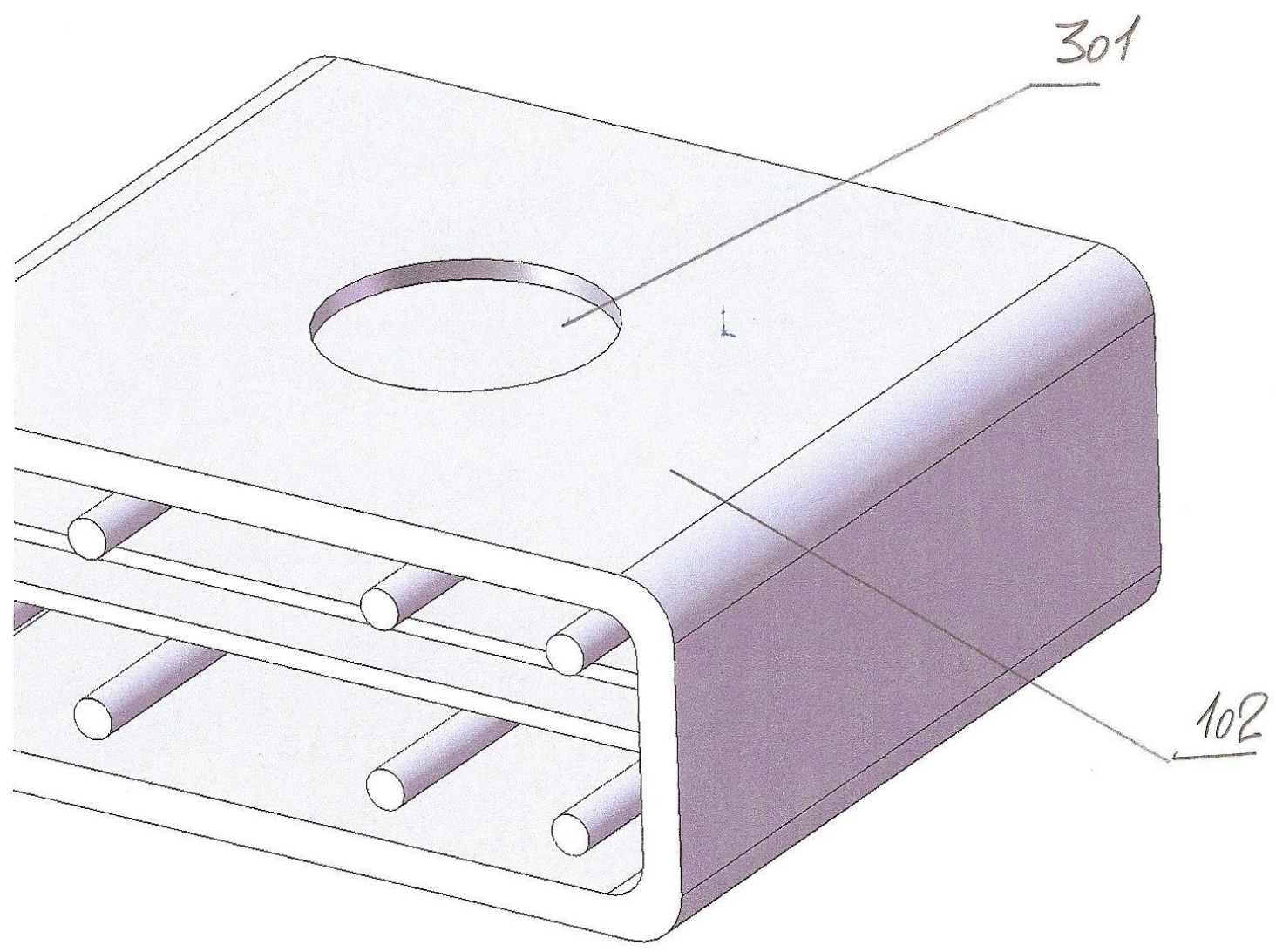

Рисунок 13, - на рисунке показан фрагмент трёхмерной модели носителя информации в районе плоского штекера с кодирующим и декодирующим диском до установки кодирующего и декодирующего диска

Цифрами на рисунке обозначены:

○ 102, - корпус плоского штекера;

○ 301, - выточка на корпусе для установки кодирующего диска в любом конструктивном варианте.

Как видно из всех представленных моделей носителя информации на рисунках 10, 11, 12 и 13, конструктивно система кодирования и декодирования довольно проста, что позволяет её интеграцию даже на носителях информации выполненных без такого устройства и таких функций.

Отсюда вытекает важная дополнительная возможность применения самых новых технологий для повышения маркетингового потенциала нового продукта. Как пример можно привести следующую технологию: 
Метод производства модуля для установки, охлаждения, управления и контроля энергетически насыщенных оптических и электронных систем включающий:

○ подготовку поверхности стальной ленты (рулона) (вместо стальной может быть указано, - металлической);

○ нанесение фоторезиста;

○ проявление фоторезиста;

○ скоростное струйное электрохимическое покрытие никелем в направленном потоке электролита (толщиной в 2-3 микрона);

○ скоростное струйное электрохимическое покрытие медью в направленном потоке электролита (толщиной в 25-35 микрон).

«Поскольку этот технологический феномен является основным базовым отличием и формирует пакет существенных преимуществ предложенного инновационного метода», - автор считает необходимым дать некоторое объяснение этому феномену.

Скоростное струйное электрохимическое покрытие - это развитый гальванический процесс в селективно ориентированном направленном потоке электролита с постоянной, обновляющей электролит системой рециркуляции электролита, в которую (систему рециркуляции) входят:

○ ёмкость с электролитом с определёнными параметрами режима содержания электролита в ёмкости такие, как:

○ концентрации никеля и меди в электролите

○ температуры электролита

○ уровня кислотности или щёлочности электролита плотности электролита

○ электрического сопротивления электролита (проводимости)

○ благодаря вышеуказанным преимуществам технологии и применяемых материалов нет необходимости в использовании органических добавок к электролиту - осветлителей;

○ насос с фильтром;

○ анод для струйной металлизации, который имеет растворимый в данном типе электролита и нерастворимый в данном типе электролита компоненты.

Компоненты, установленные последовательно по ходу движения электролита, причём нерастворимый компонент выполнен из композитной, графитной, токопроводящей ткани (типа ЭТАН). Нерастворимый компонент расположен параллельно металлизированной поверхности и последним по ходу движения электролита и первым перед покрываемой поверхностью (катодом). Оба компонента подсоединены к положительному электрическому потенциалу и также оба компонента, имеют избирательную регулируемую гидравлическую проницаемость для электролита. 
В аноде также имеется система равномерного распределения электролита по плоскости растворимого компонента, которая автоматически повторяется на нерастворимом компоненте и, следовательно и на металлизируемой поверхности - катоде:

о удаление фоторезиста;

○ травление железа, с одной стороны, на половину толщины стальной ленты

○ удаление продуктов травления с поверхности аэродинамическим и за этим, гидродинамическим воздействием;

○ опрессовка жидкотекучей полимерной композицией, - по такому технологическому порядку:

о заливка мономером

○ последующая послойная полимеризация

○ термическая стабилизация;

○ травление железа со второй стороны ленты (с теми же отличиями);

○ опрессовка со второй стороны (с теми же отличиями);

○ нанесение протектора на электропроводные структуры;

○ покрытие в вакууме всех теплопроводящих структур - слоистой системой из полупроводниковых нано-структурных поликристаллических алмазных плёнок.

Такое технологическое дополнение к традиционным технологическим приёмам и методам позволяет существенно расширить области внедрения приёмов маркетинга нового продукта.

\section{Организация корпоративных систем защиты}

Предложенная технология при организации системы защиты информационных потоков в пределах одной корпорации обеспечивает защиту на нескольких системных уровнях, включая и отслеживание в системе реального времени состояния и местонахождения каждого диска, имеющегося в корпорации.

При использовании предложенных методов кодирования, для защиты информации на мобильных внешних носителях информации, предполагается получение тех же преимуществ, что и при применении на оптических носителях и накопителях информации.

\section{Изменения в структуре и границах использования продукта, созданного в результате реализации проекта}

Таким образом на базе аналогичных решений можно создать как минимум два проекта с большим количеством аппликаций в каждом, - проект технологии для кодирования оптических накопителей информации в виде диска, включающий и соответствующее аналитически - сенсорное устройство, которое может в свою очередь иметь множество аппликаций в самых различных сферах и отраслях. 
И проект для кодирования и защиты информации на мобильных внешних носителях информации, включающий и соответствующее мобильное или стационарное сенсорное измерительно-аналитически-сравнительное устройство, также имеющее множество аппликаций и дизайн-моделей.

\section{Дополнительные устройства и системы, которые могут быть созданы на базе тех же принципиальных технологических решений}

В состав проектов при требовании потребителя проектов может быть включён раздел, касающийся дополнительных или специальных устройств, с помощью которых формируется вся корпоративная система охраны и защиты информационных потоков в пределах одной корпорации или группы корпораций или (по Российской специфике, госкорпораций) отдельных научно-исследовательских учреждений, академических институтов и крупных учреждений в системе здравоохранения.

В качестве специального продукта может быть создана система защиты информации не только в области хранения, но и в оперативной области, при передаче команд и сигналов в условиях армейских частей и соединений, и особенно, в условиях - морского флота.

В современных условиях, когда информация концентрируется в относительно очень малых размерах и объёмах устройств для её хранения, возможный ущерб от несанкционированного или преступного входа в эти массивы информации, может быть предотвращен или локализован при помощи создания специальной инфраструктуры указанных защитных систем, которая может быть стандартизована в пределах специфики данного министерства, главного управления или структурных корпоративных соединений и предприятий более низкого организационного уровня.

Серия рисунков, - от рисунка 14 до рисунка 20 показывает, как пример, применения технологий кодирования и декодирования в идентификации одноразового инструмента в лазерных медицинских приборах. 


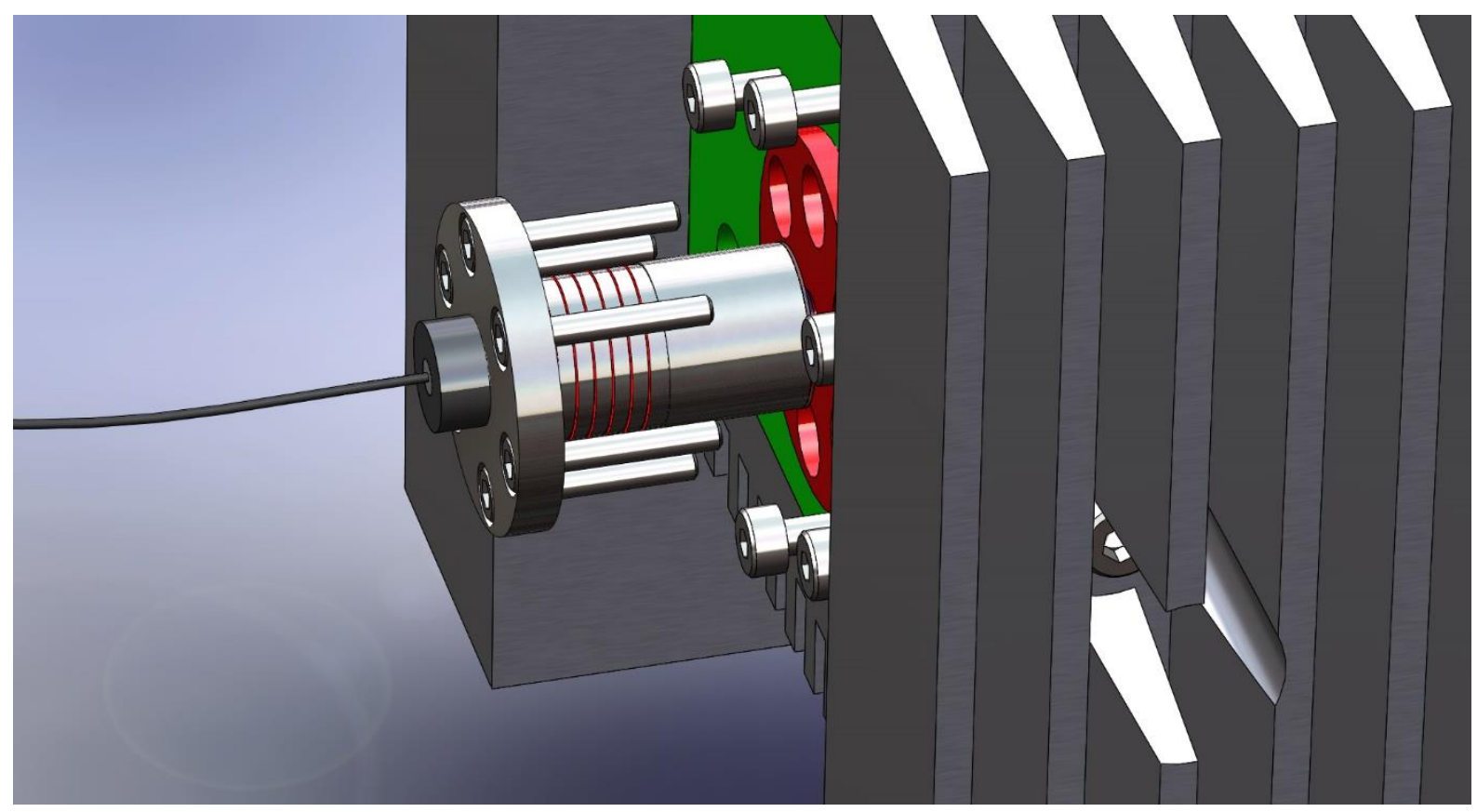

Рисунок 14, - на рисунке показана трёхмерная модель стыковочного узла лазерного эндоскопа для установки и идентификации одноразового инструмента

Такое совмещение функций повышает эффективность работы системы и существенно упрощает конструкцию узла, что в свою очередь снижает его стоимость и затраты на эксплуатацию и ремонт.

В свою очередь снижение издержек при повышении качества дают дополнительные гарантии для более уверенного маркетинга для нового продукта.

\section{Конфиденциальность информации}

Более подробно (в объёмах выходящих за пределы настоящей публикации и иллюстративных материалах к ней) вся необходимая информация может быть предоставлена при документально-юридическом формулировании намерений потенциального потребителя или партнёра, после подписания с ним договоров о конфиденциальности (по взаимно согласованной, приемлемой для обоих сторон юридической форме).

Для более полного представления о существующих физических основах выполнения операций кодирования и раскодирования оптических дисков применён магниторезонансный метод, краткое описание которого приводится ниже. 


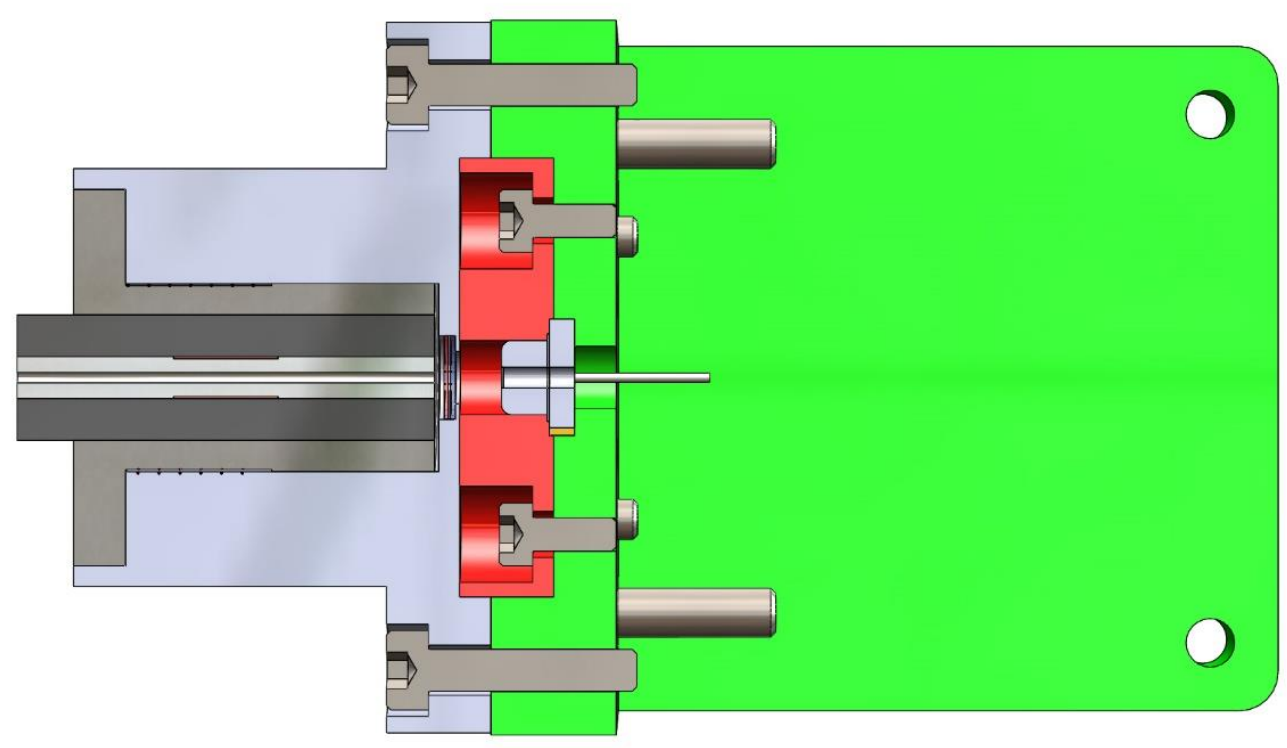

Рисунок 15, - на рисунке также показана трёхмерная модель стыковочного узла лазерного эндоскопа для установки и идентификачии одноразового инструмента.

Модель показана в осевом сечении, где видна система функиионального взаимодействия между лазерным диодом и оптическим кабелем, как осью одноразового инструмента

Система предельно проста для воспроизводства и эксплуатации

\section{Краткое описание резонансного метода}

Метод предусматривает создание переменного электромагнитного поля в пространстве, в котором располагается исследуемый образец. Это поле является посредником между резонансным контуром и испытуемым образцом.

С одной стороны, резонансный контур является эмиттером (излучателем) этого поля, а с другой - акцептором (чувствительным элементом), тех изменений в электромагнитном поле, которые вносит испытуемый образец.

Даже в отсутвии испытуемого образеца создаваемое соленоидом переменное электромагнитного поле является суммой двух электромагнитных полей, которые изменяются в противофазе друг другу.

Одно поле порождается изменением магнитной индукции соленоида и имеет своим следствием вихревое электрическое поле (Maxwell-Faraday equation). Другое порождается изменением электрического поля, созданного разностью потенциалов между крайними наиболее удалёнными друг от друга витками соленоида (если образец помещён внутрь соленоида) или разностью потенциалов между ближайшим к 
поверхности измеряемого образца витком и самим образцом (если образец расположен напротив торца соленоида), и имеет своим следствием вихревое магнитное поле (Ampère's circuital law with Maxwell's correction).

Под воздействием внешнего переменного электромагнитного поля в испытуемом образце, в зависимости от его природы, могут индуцироваться такие электрические явления, как линейные и вихревые токи проводимости, линейные и вихревые токи смещения, а также линейные и вихревые ионные токи (упорядоченное движение ионов).

В соответствии с принципом суперпозии полей эти электрические явления вносят искажения во внешнее переменное электромагнитное поле.

Эти искажения воспринимаются соленоидом резонансного датчика. Резонансный контур, в состав которого входит этот соленоид, изменяет своё поведение аналогично тому, как если бы в его состав были добавлены дополнительные элементы: конденсатор, индуктивность и резистор.

Совокупность дополнительных емкостного, индуктивного и активного сопротивлений представляет собой дополнительный импеданс, вносимый в систему испытуемым образцом, этот атрибут и измерят резонансный датчик.

Изменения параметров резонансного контура отражаются в изменении его ампдитудночастотной характеристике, а именно, меняются резонансные частота и амплитуда контура.

Исследуя эти изменения, можно судить об импедансе исследуемого образца.

\section{Принцип обработки данных, получаемых от резонансных датчиков}

Резонансный датчик позволяет определить величину суммарного импеданса исследуемого образца на рабочей частоте этого датчика (см. «Краткое описание резонансного метода»).

Сама по себе эта величина мало информативна.

Но всё коренным образом меняется, если мы имеем набор датчиков с разными рабочими частотами.

В этом случае возникает возможность использовать уникальный природный феномен, наблюдаемый во всех типах веществ: неорганических, органических и биологических. Этот феномен заключается в том, что вещество меняет свой удельный импеданс в зависимости от частоты, воздействующего на него, электрического поля и это изменение зависит от состава исследуемого вещества. 
Этот феномен исследует и активно использует быстроразвивающаяся в последнее время научное направление, называемое Магнитно-резонансная спектроскопия - impedance spectroscopy.

В англоязычных источниках её чаще называют Electrochemical Impedance Spectroscopy (EIS) (Электрохимическая Магнитно-резонансная спектроскопия - impedance spectroscopy).

Магнитно-резонансная спектроскопия - impedance spectroscopy - метод исследования различных объектов, основанный на измерении и анализе зависимостей импеданса от частоты переменного тока.

Разные объекты и процессы характеризуются разными зависимостями активного и реактивного импеданса от частоты, что делает возможным решение обратной задачи получение информации об этих объектах и процессах путем анализа частотных характеристик их отклика на переменном токе.

Тот факт, что изменение импеданса при изменении частоты зависит от состава вещества, позволяет выявить изменения и влияние каждого компонента на суммарный импеданс вещества при различных частотах.

После определения весовых коэффициентов влияния соответствующих компонентов на суммарный импеданс вещества на каждой из рабочих частот резонансных датчиков, можно на основании показаний датчиков, решая систему линейных уравнений, получить информацию о концентрации исследуемых компонентов.

На точность этого метода огромное влияние имеет правильный выбор рабочих частот датчиков.

Путём сканирования в широком диапазоне частот необходимо определить наиболее характерные для каждого компонента области частот, то есть частоты, на которых компонент даёт наибольший отклик.

Традиционная импедансная спектроскопия в своих исследованиях использует источник переменного напряжения, который контактным способом воздействует на исследуемый образец, при этом в цепи возникает электрический ток, величина и сдвиг фазы которого, зависит от импеданса образца.

Результаты отображаются, как правило, в виде фигур Лиссажу или диаграмм Найквиста. При таких исследованиях трудно добиться высокой чувствительности и точности измерений. Предлагаемая методика, в которой измерение импеданса производится с помощью резонансных контуров, обладает значительно более высокой чувствительностью и точностью, к тому же она бесконтактна. 
Существуют определённые технические трудности создания колебательного контура с перенастраиваемой в широком диапазоне резонансной частотой, поэтому для поиска «характерных» для компонентов частот придётся использовать традиционную Магнитно-резонансную спектроскопию - impedance spectroscopy.

После того, как характерные частоты будут найдены и будет созданы резонансные датчики для этих частот, созданная на базе этих датчиков система мониторинга компонентов будет обладать исключительной чувствительностью и точностью.

\section{Помехозащищённость}

Такие «механические» параметры как ВЯЗКОСТЬ, ПЛОТНОСТЬ, ПРОЗРАЧНОСТЬ, ДАВЛЕНИЕ (если среда несжимаемая) не должны оказывать никакого влияния на измеряемые электрические параметры вещества.

СКОРОСТЬ ДВИЖЕНИЯ В ТРУБОПРОВОДЕ и ТУРБУЛЕНТНОСТЬ - ЭТИ ЯВЛеНИЯ слишком медленные, чтобы оказать влияние на «мегагерцовые» процессы измерения импеданса. ЖЁСТКОСТЬ - это химический показатель, который полностью определяется входящими в вещество компонентами.

Температура, как правило, оказывает влияние на величину импеданса, но измерение температуры и её учёт при измерении импеданса не представляется сложной технической задачей.

1. Принципиальные основы защитного кодирования оптических носителей или накопителей информации, преимущественно в виде диска, прозрачного для светового потока, исходящего из выходной оптической системы одномодового лазерного диода, имеющего стандартные исполнительные размеры, - наружный диаметр, - 120 миллиметров, и толщину, - в 1,2 миллиметра.

Диск склеен из двух половин, каждая толщиной в 0,6 миллиметра; покрытие нанесено на одной из половин диска на кольце наружный диаметр которого, - 120 миллиметров, а внутренний диаметр которого, - 118 миллиметров; толщина покрытия варьируется в диапазоне от 1 микрона до 10 микрон с интервалом в 100 ангстрем.

Такая точность полностью обеспечивается свойствами и параметрами технологии скоростных электрохимических покрытий и также в эксплуатации обеспечивает высокую точность идентификации, не допуская ошибок, связанных с неточностью измерений кода.

1.1. Концептуальные основы кодирования заключаются в следующем принципе: кодирующий сигнал формируется из реакции сенсора или группы сенсоров на толщину кольцевого покрытия на диске, сравнения полученного сигнала с 
статистическим эталоном этого сигнала, - эквивалентом резонансной реакции сенсоров на толщину покрытия, удельные показатели материала покрытия, проводимости материала покрытия, плотности материала покрытия, электрического сопротивления материала покрытия.

1.2. В систему серво-маркировки отформатированного диска, которая, как правило имеет вид групповых сочетаний серво-точек на информационных треках диска, вместо одной из точек группового сочетания , вводят сигнал от декодирующего сенсора системы защитного кодирования, и, в случае совпадения интегрированного сигнала от трёх сенсоров с заданными параметрами сигнала, сервосистема дисковода начинает ориентировать фокус лазера на информационном треке, и, таким образом система начинает процесс чтения или записи на оптическом диске.

1.3. В случае несовпадения сигнала от сенсоров с статистической формой сигнала в памяти процессора дисковода, сервосистема дисковода не ориентирует и не стабилизирует траекторию фокуса луча лазерного диода на информационном треке диска и чтение или запись на диске становятся невозможными.

\section{2. ВАРИАНТЫ ИДЕНТИФИКАЦИИ ДИСКА В ДИСКОВОДЕ:}

2.1. Идентификация диска в дисководе может вестись при помощи измерения в режиме реального времени толщины покрытия, сравнения результатов измерения с хранящимся в процессоре дисковода статистическим значением этого параметра и выдачи сигнала на сравнивающее устройство в процессоре дисковода.

2.2. Процесс идентификации может вестись при вращении диска или при установке диска в дисковод.

2.3. При идентификации при установке диска в дисковод, отрицательные результаты идентификации не позволяют включение какой-либо структуры дисковода, и, наоборот положительный сигнал идентификации включает необходимые структуры дисковода.

2.4. Аналогичная логика и порядок работы имеют место и в системе кодирования и декодирования одноразового инструмента в различных приборах и технических системах любого уровня.

2.5. Такая конструктивная взаимосвязь значительно унифицирует все технологические переходы процесса идентификации, кодирования и декодирования, вне зависимости от конкретного вида и области применения продукта, а только в зависимости от конструктивных особенностей и характеристик специального узла для кодирования и декодирования. 


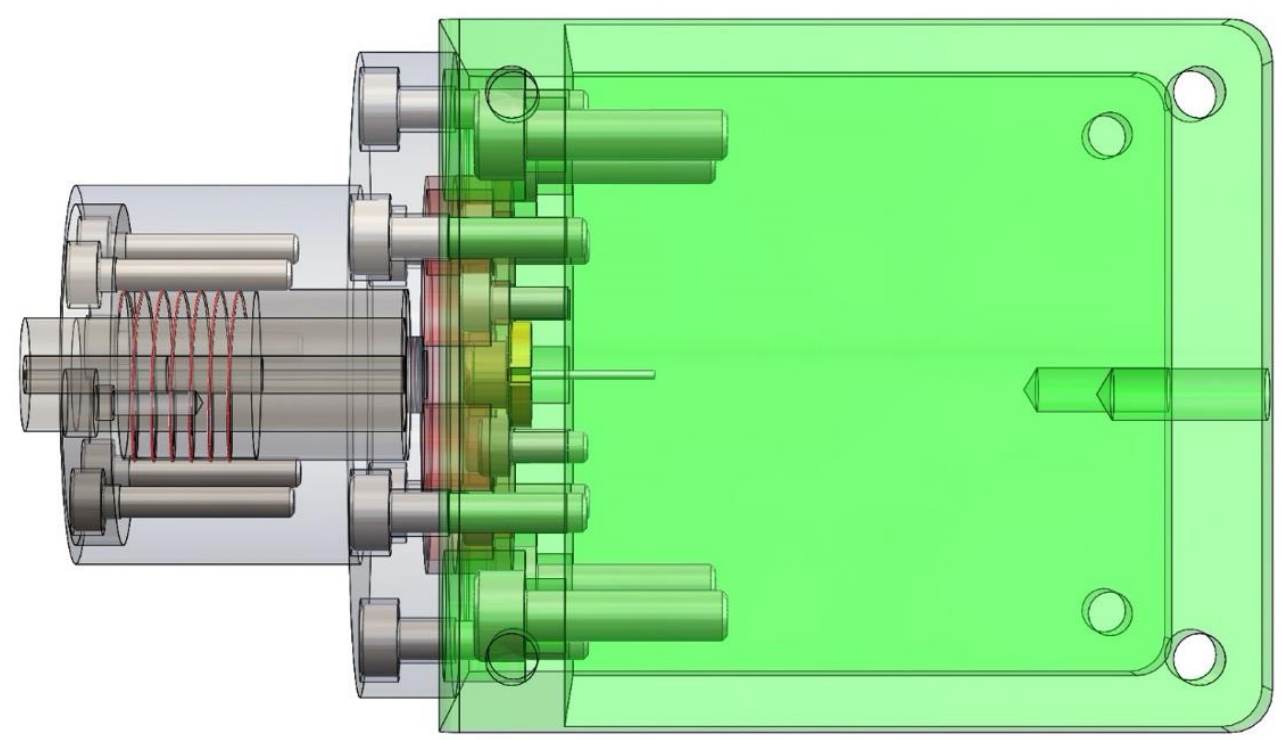

Рисунок 16, - на рисунке также показана трёхмерная модель стыковочного узла лазерного эндоскопа для установки и идентификаџии одноразового инструмента.

Модель показана в прозрачном варианте, вид сверху, где видна система функиионального взаимодействия между лазерным диодом и оптическим кабелем, как осью одноразового инструмента. Просматривается и соленоид сенсора от сигнала которого осуществляется кодирование и декодирование одноразового инструмента

\section{3. КОНСТРУКТИВНЫЕ ВАРИАНТЫ ДИСКОВОДОВ:}

3.1. Элементы защитной системы резонансного кодирования - декодирования могут без каких-либо конструктивных или схемных ограничений, быть встроены в любую существующую сегодня конструкцию дисковода, реализующую все известные технологии оптической памяти.

3.2. Существующие дисководы также могут быть модифицированы под монтаж системы микросенсоров, путём врезки сенсорного микромодуля в несущую конструкцию корпуса дисковода.

3.3. При необходимости покрытие может быть выполнено на уже существующих дисках.

4. ПРИМЕРНЫЙ ТЕХНОЛОГИЧЕСКИЙ МАРШРУТ ИЗГОТОВЛЕНИЯ ДИСКА С КОДИРУЮЩИМ ПОКРЫТИЕМ:

4.1. Для изготовления оптического диска с защитным кодирующим покрытием не требуется специальных технологий и оборудования.

4.2. Для изготовления может быть использовано модернизированное технологическое оборудование, которое используется в настоящее время. 
4.3. Нанесение кодирующего покрытия можно совместить с изготовлением копии диска в прессформе с использованием мастер-диска с идентификационной точкой в отформатированной системе сервомаркировки, которые таким образом будут отпечатаны на каждом информационном треке, - а их в обычном оптическом диске более 37000.

\section{5. ВАРИАНТЫ ИСПОЛЬЗОВАНИЯ ДИСКОВ С ЗАЩИТНЫМ ПОКРЫТИЕМ В СИСТЕМАХ ОПТИЧЕСКОЙ ПАМЯТИ КОРПОРАТИВНЫХ КЛИЕНТОВ:}

5.1. Примерная схема использования дисков с защитным кодированиемдекодированием у корпоративных клиентов предусматривает изготовление для каждого такого клиента определённого количества дисков с присущими только для этого клиента параметрами толщины и координатами микро - сенсоров.

5.2. Конструкция и техническая характеристика сенсорного микромодуля также может быть модернизирована исходя из пожеланий клиента, но в соответствии с контрольными параметрами защитного кодирующего покрытия на дисках.

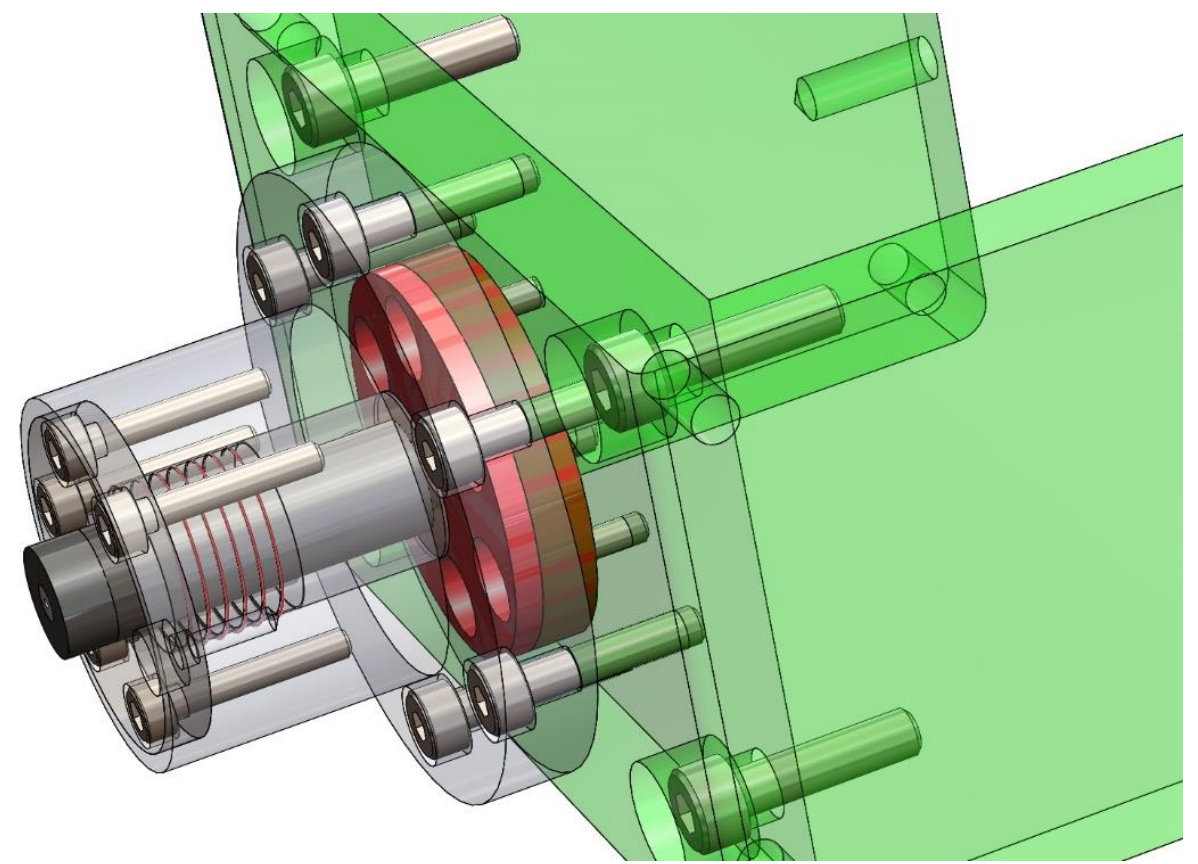

Рисунок 17, - на рисунке также показана трёхмерная модель стыковочного узла лазерного эндоскопа для установки и идентификаџии одноразового инструмента.

Модель показана в прозрачном варианте, вид в изометрии, где видна система функционального взаимодействия между лазерным диодом и оптическим кабелем, как осью одноразового инструмента. Просматривается и соленоид сенсора от сигнала которого осуществляется кодирование и декодирование одноразового инструмента 


\section{6. ВАРИАНТЫ ИСПОЛЬЗОВАНИЯ ДИСКОВ С ЗАЩИТНЫМ КОДИРОВАНИЕМ В СИСТЕМАХ БЫТОВОЙ РАДИОТЕХНИКИ:}

6.1. Диски с защитным кодированием могут быть использованы в системах Blu-Ray и HD DVD.

Кроме этого, система защитного кодирования может быть применена в новых разработках и технологиях оптической цифровой памяти в том числе и дисках с особо высокой плотностью записи, многослойных дисках, монолитных оптических дисках с объёмом памяти в 1 и более терабайт.

6.2. При изготовлении дисков, необходимую индикацию в серво-маркировку, можно вносить в процессе прессования.

Сервопривод дисковода начинает ориентацию фокусной точки лазерного луча только при совпадении кодирующего сигнала от системы кодирования и декодирования, сформированного системой из трёх микро-сенсоров, которые при помощи методов магнитного резонанса, сравнивают толщину покрытия с эталоном и при совпадении параметров сигнала с эталоном хотя бы у двух сенсоров, добавляют полученный сигнал в систему символов и маркирующих точек серво-маркировки, считывая которые сервопривод дисковода начинает стабилизировать фокус лазера на необходимом треке на поле записи диска.

\section{7. ВАРИАНТЫ ИСПОЛЬЗОВАНИЯ ДИСКОВ С ЗАЩИТНЫМ ПОКРЫТИЕМ В ПЕРСОНАЛЬНЫХ КОМПЬЮТЕРАХ:}

7.1. Технология изготовления дисков для персональных компьютеров аналогична технологии изготовления такого рода дисков для других вариантов оптической памяти.

7.2. Методика использования дисков с защитным кодированием формируется исходя из типа компьютера, степени его насыщенности и мощности, быстродействия и Т.П.

7.3. Особо важным становится возможность использования техники и технологии защитного кодирования в создаваемых гибридных дисках, сочетающих в себе жёсткий диск с оптическим диском. 


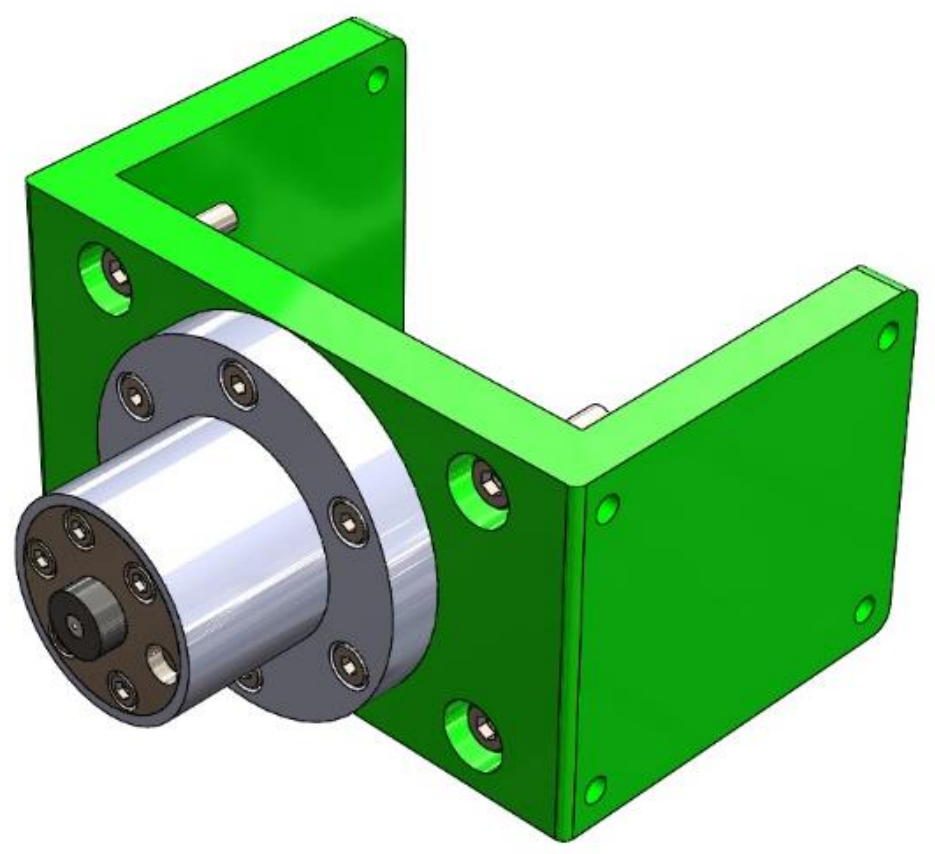

Рисунок 18, - на рисунке показана трёхмерная модель корпуса штекерного разъёма пристыкованного к основной несущей лазерный диод детали (на рисунке обозначена зелёным иветом)

\section{Предложение по системе получения профессиональной информации из интернета}

В качестве основного инструмента выступает оптический диск, на котором нанесено кодирующее покрытие в кольцевой зоне, в которой нет информационной записи.

В качестве вспомогательного инструмента выступает микросенсор, который встраивается в дисковод.

Сигнал от микросенсора формируется при измерении толщины покрытия; точность измерения, - 100 ангстрем и это величина на которую отличается каждая группа дисков от другой группы.

Сигнал от микросенсора является кодом для входа в массивы информации, размещённые в интернете.

Программное обеспечение должно давать возможность идентифицировать сигнал от микросенсора и в случае совпадения сигнала с эталонным открывать массивы информации и в процессе её скачивания продолжать контролировать достоверность сигнала до завершения процесса скачивания информации. 
Это даёт возможность предотвратить замену диска во время записи на нелицензированный.

Подделать такой диск невозможно, так как толщина покрытия определяется при изготовлении и, даже имея такой диск, невозможно им воспользоваться, без микросенсора, настроенного на строго определённый характер сигнала.

Диски и сенсоры могут выпускаться на любом сегодня существующем производстве оптических дисков; диски могут выпускаться сериями по $100-250$ штук с одинаковой толщиной кодирующей ленты и с комплектом сенсоров.

Каждый пользователь может приобрести одну или несколько серий дисков и использовать их при работе с интернетом.

По такому же принципу программы и другая информация могут рассылаться пользователям, только в обратном порядке, что гарантирует полную конфиденциальность и защиту при нахождении в интернете от несанкционированных посланий и вирусов.

Это конечно очень общая информация, если Вы читатели - сочтёте её заслуживающей внимания, то автор мог бы детализировать этот проект.

Ввиду того, что механическая часть этого проекта в принципе реализована, этот проект, - это программное обеспечение, что может быть станет основой проекта в этом направлении.

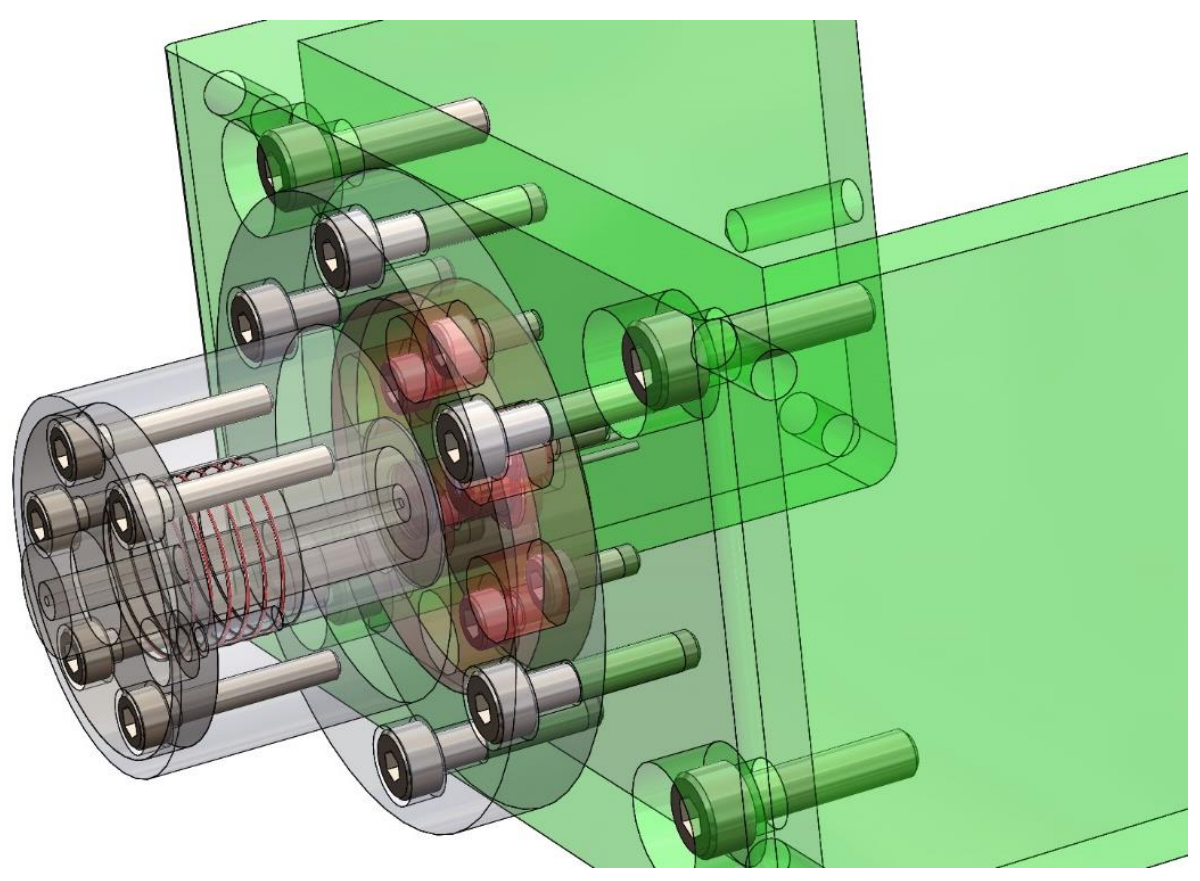


Рисунок 19, - на рисунке показан коаксиальный корпус штекерного разъёма с кодирующим и декодирующим устройством в виде коаксиально установленного соленоида, который совместно с импульсным генератором формирует кодирующий или декодирующий сигнал и после его прохождения сортирует и передаёт на аналитический блок резонансный сигнал

\section{Преимущества предложенной технологии, отвечающей на существо проблем, выявленных на рынке систем оптических носителей информации:}

1. Имеется множество вариантов толщин кодирующих покрытий, которые позволяют иметь множество вариантов защитного кода, в отличие от известных технологий, которые имеют только один вариант кода.

2. В процессе нанесения покрытия применяется технология контроля полностью идентичная технологии декодирования, что позволяет полностью контролировать качество кодирования в процессе изготовления диска, без удаления диска с конвейера, в отличие от существующих технологий, в которых диск для контроля необходимо удалять с конвейера и устанавливать в контрольное приспособление.

Таким образом контроль выборочный, а в предложенной технологии , - 100\% контроль, что исключает выпуск бракованных дисков, которые в существующих технологиях обнаруживаются только во время эксплуатации.

3. В предложенной технологии имеется возможность кодирования всех категорий и типов дисков вне зависимости от формата записи и чтения, в отличие от существующих технологий, в которых кодирование зависит от формата записи и чтения диска.

4. В предложенной технологии кодирующее покрытие может служить основанием для персонального секретного кода или шифра, чего нет в существующих технологиях.

5. В предложенной технологии сенсор декодирования и идентификации является мобильным и может иметь несколько вариантов поставки, в том числе и автономный вариант, не связанный с дисководом, а в существующих технологиях система декодирования устанавливается только в дисководах; таким образом контролировать наличие и правильность кодирования можно только в процессе установки диска в дисковод, а в предложенной технологии контролировать и идентифицировать код можно вне дисковода, например в магазинах или на проходных предприятий и учреждений, что особенно важно для обеспечения полного режима конфиденциальности информации.

6. В предложенной технологии декодирование исключает какую либо зависимость от оптических систем дисковода, но результаты декодирования могут изменить работу 
оптических систем, например серво - привода для ориентации и контроля положения фокуса читающего или записывающего лазера, в отличие от существующих технологий, в которых процесс декодирования полностью зависит от оптических элементов дисковода, что усложняет его конструкцию и резко снижает надёжность.

7. Предложенная технология имеет несколько иерархий принципиальной рабочей схемы, имеет гибкий алгоритм и может быть встроена в любую охранную систему оптической памяти в том числе и в гибридные носители информации, имеющие кроме оптического компонента и носители, построенные на других базовых принципах; существующие технологии не обладают указанной гибкостью.

8. Предложенная технология позволяет использовать код диска как вводный пароль для входа в профессиональные массивы информации интернета, чем не обладают существующие технологии.

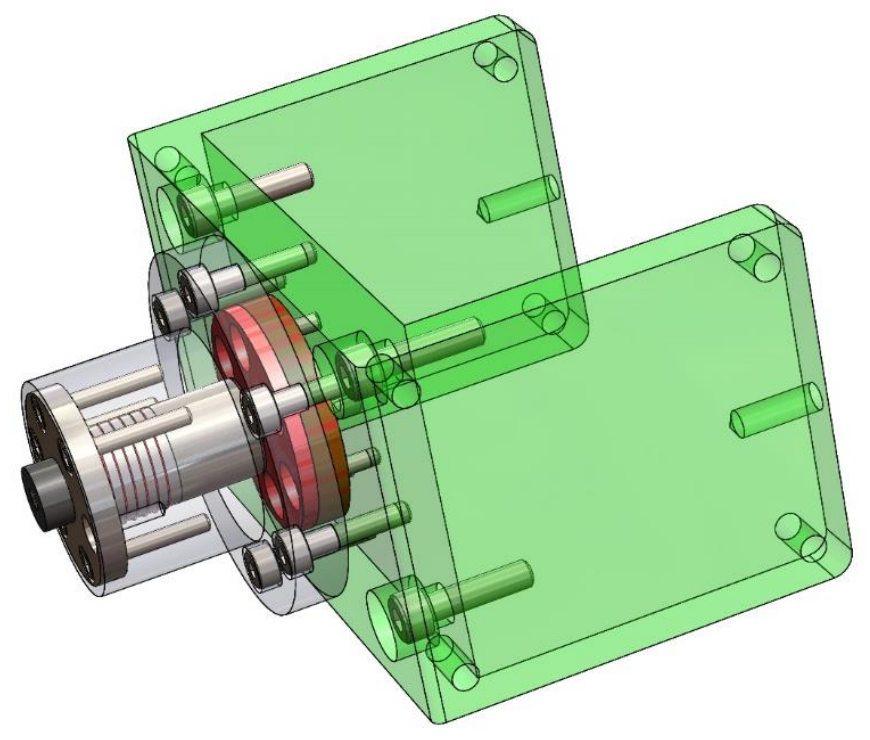

Рисунок 20, - на рисунке показан модуль лазерного диода с кодирующей и декодирующей втулкой, содержащей сенсор-соленоид, расположенный коаксиально к штекеру одноразового инструмента, причём штекер выполнен в виде втулки, толщина стенки которой является ключом к иифрующему коду, который идентифицируют при помощии резонансной спектроскопии

Технологическое и конструктивное решение такого интегративного сенсора, позволяет его встраивание в практически любую техническую систему, где подсоединение, например одноразового инструмента, ведётся при помощи штекерного разъёма. 
По результатам комплексного анализа с применением алгоритмов патентной и лицензионной стратегии, предварительно разрабатывается следующая общая стратегия маркетинга.

Предварительное определение базового продукта проекта и его функциональных и эксплуатационных особенностей и характеристик:

1. Основной сектор рынка, - корпоративные клиенты:

○ Банки и финансовые компании;

○ Промышленные корпорации;

○ Научно - исследовательские лаборатории;

○ Транспортные компании, вокзалы, аэропорты и морские порты;

○ Крупные торговые сети;

○ Муниципальные службы;

○ Правительственные организации и учреждения;

○ Крупные медицинские учреждения;

○ Страховые компании;

○ Рода войск вооружённых сил;

○ Полиция и спецслужбы.

2. Характеристика продукта (как пример):

○ Продукт проекта (основной), - оптический диск с кодирующим кольцом и дисковод с встроенным сенсорным модулем, как правило состоящим из трёх микросенсоров;

○ В случае необходимости сенсорный модуль может поставляться без дисковода;

○ В случае необходимости компания, ведущая проект, может предоставлять услуги для корпоративных клиентов, организовывая ввод в действие системы кодирования и защиты информации, - под ключ;

\section{СПИСОК ИСПОЛЬЗОВАННОЙ ЛИТЕРАТУРЫ, ПАТЕНТНОЙ И ЛИЦЕНЗИОННОЙ ИНФОРМАЦИИ}

\section{ПРИЛОЖЕНИЕ 1}

United States Patent Application

20190281321

Kind Code

ZHAO; Xin ; et al.

September 12, 2019 


\title{
METHOD AND APPARATUS FOR VIDEO CODING
}

\begin{abstract}
Aspects of the disclosure provide method and apparatus for video coding. In some examples, an apparatus includes processing circuitry. The processing circuitry determines an intra prediction mode for generating a prediction image of a current block, determines one or more primary transforms according to signaling information that is extracted from a coded video bitstream, and determines a secondary transform according to the determined intra prediction mode and the determined one or more primary transforms. The processing circuitry also reconstructs a residue image of the current block based on one or more coefficients of an input block extracted from the coded video bitstream, the determined one or more primary transforms, and the determined secondary transform. The processing circuitry then reconstructs an image of the current block based on the prediction image and the residue image of the current block.
\end{abstract}

\section{ПРИЛОЖЕНИЕ 2}

United States Patent Application

20190281270

Kind Code

CHEN; Ying ; et al.

September 12, 2019

SHARING OF MOTION VECTOR IN 3D VIDEO CODING

\begin{abstract}
Joint coding of depth map video and texture video is provided, where a motion vector for a texture video is predicted from a respective motion vector of a depth map video or vice versa. For scalable video coding, depth map video is coded as a base layer and texture video is coded as an enhancement layer(s). Inter-layer motion prediction predicts motion in texture video from motion in depth map video. With more than one view in a bitstream (for multiview coding), depth map videos are considered monochromatic camera views and are predicted from each other. If joint multiview video model coding tools are allowed, inter-view motion skip is used to predict motion vectors of texture images from depth map images. Furthermore, scalable multiview coding is utilized, where inter-view prediction is applied between views in the same dependency layer, and inter-layer (motion) prediction is applied between layers in the same view.
\end{abstract}

\section{ПРИЛОЖЕНИЕ 3}

United States Patent Application

20190273948

Kind Code

Yin; Hujun ; et al.

September 5, 2019 


\title{
METHOD AND SYSTEM OF NEURAL NETWORK LOOP FILTERING FOR VIDEO CODING
}

\begin{abstract}
A method, system, medium, and article provide neural network loop filtering for video coding with multiple alternative neural networks.

ПРИЛОЖЕНИЕ 4

United States Patent Application

20190273932

Kind Code

A1

HAQUE; MUNSI ; et al.

September 5, 2019

VIDEO CODING SYSTEM WITH TEMPORAL SCALABILITY AND METHOD OF

OPERATION THEREOF
\end{abstract}

\begin{abstract}
A method of operation of a video coding system includes: receiving a video bitstream; extracting a video syntax from the video bitstream; extracting a hypothetical reference decoder (HRD) fixed syntax from the video syntax; extracting a HRD variable syntax from the video syntax; extracting a temporal layer from the video bitstream based on the HRD fixed syntax and the HRD variable syntax; and forming a video stream based on the temporal layer for displaying on a device.
\end{abstract}

\section{ПРИЛОЖЕНИЕ 5}

United States Patent Application

20190268098

Kind Code

A1

CHUN; Jinyoung ; et al.

August 29, 2019

DATA TRANSMISSION METHOD IN WIRELESS COMMUNICATION SYSTEM AND DEVICE THEREFOR

\begin{abstract}
A downlink (DL) multi-user (MU) transmission method in a wireless local area network (WLAN) system, the DL MU transmission method including receiving a DL MU physical protocol data unit (PPDU) including a physical preamble and a data field from STA (Station) and transmitting the ACK frames in response to the DL MU PPDU to STA. In addition, the data field includes at least one medium access control (MAC) protocol data unit (MPDU), the
\end{abstract}


at least one MPDU includes a MAC header and a MAC frame body, wherein the MAC header includes acknowledge (ACK) indication information, the ACK indication information includes frequency resource allocation information for an uplink (UL) MU orthogonal frequency division multiple access (OFDMA) transmission of ACK frames and modulation and coding scheme (MCS) level information, and the frequency resource allocation information includes an index value indicating a resource unit allocated for the UL MU OFDMA transmission of the ACK frames, and the resource unit corresponds to a 26-tone resource unit, a 52-tone resource unit, a 106-tone resource unit, a 242-tone resource unit, a 484-tone resource unit, or a 996-tone resource unit.

\title{
ПРИЛОЖЕНИЕ 6
}

United States Patent Application

Kind Code

JANSSON TOFTG RD; Tomas ; et al.
20190268016

A1

August 29, 2019

\section{METHODS AND DEVICES FOR VECTOR SEGMENTATION FOR CODING}

\begin{abstract}
A method for partitioning of input vectors for coding is presented. The method comprises obtaining of an input vector. The input vector is segmented, in a non-recursive manner, into an integer number, N.sup.SEG, of input vector segments. A representation of a respective relative energy difference between parts of the input vector on each side of each boundary between the input vector segments is determined, in a recursive manner. The input vector segments and the representations of the relative energy differences are provided for individual coding. Partitioning units and computer programs for partitioning of input vectors for coding, as well as positional encoders, are presented.
\end{abstract}

\section{ПРИЛОЖЕНИЕ 7}

United States Patent Application

20190261010

Kind Code

A1

Luo; Ning ; et al.

August 22, 2019

METHOD AND SYSTEM OF VIDEO CODING WITH REDUCED SUPPORTING DATA SIDEBAND BUFFER USAGE

\begin{abstract}
Methods, systems, and articles of video coding with reduced supporting data sideband buffer usage.
\end{abstract}




\title{
ПРИЛОЖЕНИЕ 8
}

United States Patent Application

20190253706

Kind Code

ZHAO; Liang ; et al.

August 15, 2019

METHOD AND APPARATUS FOR USING AN INTRA PREDICTION CODING TOOL FOR INTRA PREDICTION OF NON-SQUARE BLOCKS IN VIDEO COMPRESSION

\begin{abstract}
A method for video decoding includes determining whether an angular intra prediction mode for a current block is a wide angle mode that is spaced apart from a vertical mode and a horizontal mode by an angular distance that is more than a predetermined threshold. The method further includes, in response to the determination that the angular intra prediction mode is the wide angle mode, determining a non-wide angle mode that corresponds to the wide angle mode, the non-wide angle mode being spaced apart from the vertical mode or the horizontal mode by an angular distance that is less than or equal to the predetermined threshold. The method includes determining whether a coding tool is specified for the determined corresponding non-wide angle mode, and, in response to the determination that the coding tool is specified for the determined corresponding non-wide angle mode, using the coding tool in a decoding process.
\end{abstract}

\section{ПРИЛОЖЕНИЕ 9}

United States Patent Application

20190181979

Kind Code

Wang; Ying ; et al.

June 13, 2019

ADJUSTED FRACTALLY ENHANCED KERNEL POLAR CODES FOR ACHIEVABLE SIGNAL-TO-NOISE RATIO SPIKE MITIGATION

\begin{abstract}
Methods, systems, and devices for wireless communications are described. In some systems, wireless devices may implement adjusted fractally enhanced kernel polar coding. An encoder may receive a number of information bits and a block size for transmission, and may append an additional number of information bits to the information bits for transmission. The encoder may perform a recursive bit allocation process to allocate the aggregate set of information bits between a set of sub-blocks based on mutual information metrics. To obtain the correct number of information bits and block size, the encoder may remove a number of information bits equal to the number of appended additional bits (e.g., from a first half of the sub-blocks), assign the remaining information bits to bit channels in each sub-block, and block puncture a set of bits
\end{abstract}


(e.g., from the first half). The resulting codeword may mitigate occurrences of achievable signal-to-noise ratio (SNR) spikes.

\title{
ПРИЛОЖЕНИЕ 10
}

United States Patent Application

20190182503

Kind Code

Tsai; Yi-Ting ; et al.

June 13, 2019

METHOD AND IMAGE PROCESSING APPARATUS FOR VIDEO CODING

\begin{abstract}
A method and an image processing apparatus for video coding are proposed. The method is applicable to an image processing apparatus and includes the following steps. A current coding unit is received, and the number of control points of a current coding unit is set, where the number of control points is greater than or equal to 3. At least one affine model is generated based on the number of control points, and an affine motion vector corresponding to each of the at least one affine model is computed. A motion vector predictor of the current coding unit is computed based on the at least one motion vector so as to accordingly perform interprediction coding on the current coding unit.
\end{abstract}

\section{ПРИЛОЖЕНИЕ 11}

United States Patent Application

20190190578

Kind Code

A1

Mittal; Udar ; et al.

June 20, 2019

CODING MAIN BEAM INFORMATION IN CSI CODEBOOK

\begin{abstract}
Apparatuses, methods, and systems are disclosed for preparing a channel state information ("CSI") codeword. One apparatus includes a processor and a transceiver configured to communicate 805 with a transmit-receive point ("TRP") over a radio access network using spatial multiplexing, wherein multiple transmission layers are transmitted at a time, each transmission layer comprising multiple beams. The processor identifies a main beam for each of the multiple transmission layers and determines whether the main beams of each transmission layer are the same. The processor prepares a CSI codeword, wherein the CSI codeword comprises a first bit indicating whether the main beams of each transmission layer are the same, a first set of bits coding the main beams, and a second set of bits coding the remaining beams. The transceiver transmits the CSI codeword to the TRP.
\end{abstract}


ПРИЛОЖЕНИЕ 12

United States Patent Application

20190200025

Kind Code

A1

Li; Bin ; et al.

June 27, 2019

\title{
CODED-BLOCK-FLAG CODING AND DERIVATION
}

\begin{abstract}
Techniques for coding and deriving (e.g., determining) one or more coded-block-flags associated with video content are described herein. A coded-block-flag of a last node may be determined when coded-block-flags of preceding nodes are determined to be a particular value and when a predetermined condition is satisfied. In some instances, the predetermined condition may be satisfied when log.sub.2(size of current transform unit) is less than log.sub.2 (size of maximum transform unit) or log.sub.2(size of current coding unit) is less than or equal to $\log$.sub.2(size of maximum transform unit)+1. The preceding nodes may be nodes that precede the last node on a particular level in a residual tree.
\end{abstract}

\section{ПРИЛОЖЕНИЕ 13}

United States Patent Application

20190222863

Kind Code

A1

HANNUKSELA; Miska Matias ; et al.

July 18, 2019

VIDEO CODING AND DECODING

\begin{abstract}
There is disclosed a method, an apparatus, a server, a client and a non-transitory computer readable medium comprising a computer program stored therein for multi view video coding and decoding. View random access (VRA) pictures or access units are coded into a multiview bitstream. VRA pictures enable starting the decoding of a subset of the views present in the bitstream. The views selected to be accessible in VRA pictures are alternated in successive VRA pictures so that all views are gradually reconstructed when two or more VRA pictures have been decoded.
\end{abstract}

\section{ПРИЛОЖЕНИЕ 14}

United States Patent Application

20190200026

Kind Code

LAINEMA; Jani ; et al.

June 27, 2019 
APPARATUS, A METHOD AND A COMPUTER PROGRAM FOR VIDEO CODING

\begin{abstract}
There is disclosed an apparatus, a method and a computer program for video coding. The apparatus comprises a selector configured for selecting a pixel for prediction; a projection definer configured for determining a projection of said pixel to a set of reference pixels; and a prediction definer configured for selecting one or more reference pixels from said set of reference pixels on the basis of said projection, and using said selected one or more reference pixels to obtain a prediction value for said pixel to be predicted.
\end{abstract}

ПРИЛОЖЕНИЕ 15

United States Patent Application

20190208222

Kind Code

UGUR; Kemal ; et al.

July 4, 2019

APPARATUS, A METHOD AND A COMPUTER PROGRAM FOR VIDEO CODING AND DECODING

\begin{abstract}
There are disclosed various methods, apparatuses and computer program products for video encoding and decoding. In other embodiments, there is provided a method, an apparatus, a computer readable storage medium stored with code thereon for use by an apparatus, and a video encoder, for encoding a scalable bitstream, to provide indicating an encoding configuration, where only samples and syntax from intra coded pictures of base layer is used for coding the enhancement layer pictures. In other embodiments, there is provided an apparatus, a computer readable storage medium stored with code thereon for use by an apparatus, and a video decoder, for decoding a scalable bitstream, to receive indications of an encoding configuration, where only samples and syntax from intra coded pictures of base layer is used for coding the enhancement
\end{abstract}

\title{
ПРИЛОЖЕНИЕ 16
}

United States Patent Application

20190222859

Kind Code

CHUANG; Tzu-Der ; et al.

July 18, 2019

METHOD AND APPARATUS OF CURRENT PICTURE REFERENCING FOR VIDEO CODING 


\begin{abstract}
A method and apparatus for a video coding system with the current picture referencing (CPR) mode enabled are disclosed. According to one method, the luma and chroma blocks of the current image are jointly coded using a same coding unit (CU) structure if the CPR mode is selected for the luma and the chroma blocks. Alternatively, if the luma and chroma components are partitioned into the luma and the chroma blocks separately using separate CU structures, the luma and chroma blocks are encoded or decoded using a coding mode selected from a coding mode group excluding the CPR mode. According to another method, the luma and chroma blocks of the current image are coded separately using a different CU structure if the CPR mode is selected for the luma and chroma blocks. In yet another method, reconstructed reference data is disclosed for the CPR mode with CU equal to PU.
\end{abstract}

\title{
ПРИЛОЖЕНИЕ 17
}

United States Patent Application

20190230365

Kind Code

Tanner; Jason ; et al.

July 25, 2019

VIDEO CLUSTER ENCODING FOR MULTIPLE RESOLUTIONS AND BITRATES WITH PERFORMANCE AND QUALITY ENHANCEMENTS

\begin{abstract}
Techniques related to video cluster encoding are discussed. Such techniques include encoding the video at a first resolution and first bitrate, translating block based coding parameters corresponding thereto to block based encode controls for encode of the same video at a second resolution or a second bitrate, and encoding the video at the second resolution and/or bitrate using the encode controls.
\end{abstract}

ПРИЛОЖЕНИЕ 18

United States Patent Application

20190246126

Kind Code

Abbas; Adeel ; et al.

August 8, 2019

APPARATUS AND METHODS FOR VIDEO COMPRESSION USING MULTIRESOLUTION SCALABLE CODING

\section{Abstract}


Apparatus and methods for digital video data compression via a scalable, multi-resolution approach. In one embodiment, the video content may be encoded using a multi-resolution and/or multi-quality scalable coding approach that reduces computational and/or energy load on a client device. In one implementation, a low fidelity image is obtained based on a first full resolution image. The low fidelity image may be encoded to obtain a low fidelity bitstream. A second full resolution image may be obtained based on the low fidelity bitstream. A portion of a difference image obtained based on the second full resolution image and the first full resolution may be encoded to obtain a high fidelity bitstream. The low fidelity bitstream and the high fidelity bitstream may be provided to e.g., a receiving device.

\title{
ПРИЛОЖЕНИЕ 19
}

United States Patent Application

20190246118

Kind Code

A1

YE; Jing ; et al.

August 8, 2019

METHOD AND APPARATUS FOR VIDEO CODING IN MERGE MODE

\begin{abstract}
A method for video coding using a merge mode by a decoder or encoder. An embodiment of the method includes receiving a current block having a block size, setting a grid pattern based on the block size of the current block, wherein the grid pattern partitions a search region adjacent to the current block into search blocks, and a size of the search blocks is determined according to the block size of the current block, and searching for one or more spatial merge candidates from candidate positions in the search blocks to construct a candidate list that includes the one or more spatial merge candidates.
\end{abstract}

\section{ПРИЛОЖЕНИЕ 20}

United States Patent Application

20190034583

Kind Code

A1

Kartalov; Emil P. ; et al.

January 31, 2019

SIGNAL ENCODING AND DECODING IN MULTIPLEXED BIOCHEMICAL ASSAYS

\begin{abstract}
This disclosure provides methods, systems, compositions, and kits for the multiplexed detection of a plurality of analytes in a sample. In some examples, this disclosure provides methods, systems, compositions, and kits wherein multiple analytes may be detected in a single sample volume by acquiring a cumulative measurement or measurements of at least one quantifiable component of a signal. In some cases, additional components of a signal, or
\end{abstract}


additional signals (or components thereof) are also quantified. Each signal or component of a signal may be used to construct a coding scheme which can then be used to determine the presence or absence of any analyte.

\title{
ПРИЛОЖЕНИЕ 21
}

United States Patent Application

20170236521

Kind Code

Chebiyyam; Venkata Subrahmanyam

Chandra Sekhar ; et al.

August 17, 2017

\section{ENCODING OF MULTIPLE AUDIO SIGNALS}

\begin{abstract}
A device includes an encoder and a transmitter. The encoder is configured to determine a mismatch value indicative of an amount of temporal mismatch between a reference channel and a target channel. The encoder is also configured to determine whether to perform a first temporal-shift operation on the target channel at least based on the mismatch value and a coding mode to generate an adjusted target channel. The encoder is further configured to perform a first transform operation on the reference channel to generate a frequency-domain reference channel and perform a second transform operation on the adjusted target channel to generate a frequency-domain adjusted target channel. The encoder is also configured to estimate one or more stereo cues based on the frequency-domain reference channel and the frequency-domain adjusted target channel. The transmitter is configured to transmit the one or more stereo cues to a receiver.
\end{abstract}

